\title{
DSC-Ritz method for the free vibration analysis of Mindlin plates
}

\author{
Yunshan $\mathrm{Hou}^{1}$, G. W. Wei ${ }^{2,3, *, \dagger}$ and Y. Xiang ${ }^{4}$ \\ ${ }^{1}$ Department of Computational Science, National University of Singapore, Singapore 117543 \\ ${ }^{2}$ Department of Mathematics, Michigan State University, East Lansing, MI 48824, U.S.A. \\ ${ }^{3}$ Department of Electrical and Computer Engineering, Michigan State University, East Lansing, \\ MI 48824, U.S.A. \\ ${ }^{4}$ School of Engineering and Industrial Design and Centre for Construction Technology and Research, \\ University of Western Sydney, Penrith South DC, NSW 1797, Australia
}

\begin{abstract}
SUMMARY
This paper introduces a novel method for the free vibration analysis of Mindlin plates. The proposed method takes the advantage of both the local bases of the discrete singular convolution (DSC) algorithm and the $p b-2$ Ritz boundary functions to arrive at a new approach, called DSC-Ritz method. Two basis functions are constructed by using DSC delta sequence kernels of the positive type. The energy functional of the Mindlin plate is represented by the newly constructed basis functions and is minimized under the Ritz variational principle. Extensive numerical experiments are considered by different combinations of boundary conditions of Mindlin plates of rectangular and triangular shapes. The performance of the proposed method is carefully validated by convergence analysis. The frequency parameters agree very well with those in the literature. Numerical experiments indicate that the proposed DSC-Ritz method is a very promising new method for vibration analysis of Mindlin plates. Copyright (C) 2004 John Wiley \& Sons, Ltd.
\end{abstract}

KEY WORDS: DSC kernel; Ritz method; meshless method; Mindlin plate theory; vibration analysis

\section{INTRODUCTION}

Plates are some of the most important structural elements and its theoretical descriptions were established by Chladni [1] and Kirchhoff [2]. The classical thin (Kirchhoff) plate theory has limited success for thick plates because no account is taken for the effect of transverse shear deformation on the mechanical behaviour of thick plates [3]. To allow for this effect, Mindlin [4] proposed a simple model by assuming a constant transverse shear-strain distribution through the plate thickness. Analytical solution to Mindlin plates is scarce $[5,6]$, and numerical computations are indispensable for obtaining approximate solutions that are important in engineering practices.

Typically, structural computations are accomplished by using either global methods or local methods. Global methods, such as the Rayleigh method [7], Ritz methods [8-14], series

*Correspondence to: G. W. Wei, Department of Mathematics, Michigan State University, East Lansing, MI 48824, U.S.A.

†E-mail: wei@math.msu.edu

Received 23 April 2003

Copyright (c) 2004 John Wiley \& Sons, Ltd.

Revised 16 January 2004

Accepted 7 May 2004 
expansion [15], methods of differential quadrature [16] and generalized differential quadrature $[17,18]$, etc, are highly accurate but are often cumbersome to implement in dealing with complex geometries and non-conventional boundary conditions. In contrast, local methods, such as finite strip methods [19] and spline finite strip methods [20], are easy to implement for complex geometries and discontinuous boundary conditions. However, the accuracy of local methods is relatively low compared to that of global methods. As point out by Zienkiewicz, local methods might converge slowly and are too expensive for the prediction of short waves (i.e., high-order eigenmodes) [21].

Recently, the discrete singular convolution (DSC) algorithm [22] has emerged as a novel approach that exhibits global methods' accuracy for integration and local methods' flexibility for handling complex geometries and boundary conditions. The mathematical foundation of the DSC algorithm is the theory of distributions and wavelet analysis. The same theory also underpins the basis for singular transforms of Hilbert type, Abel type and delta type. These transforms have important applications to analytical signal processing, tomography and surface interpolation. Numerical solutions to differential equations are formulated via the singular kernels of the delta type. Based on the DSC framework, a unification was discussed for a number of conventional computational methods, including global, local, Galerkin, collocation, and finite difference methods [23,24].

The DSC algorithm has found its success in fluid dynamic simulation [25] and electromagnetic wave propagation [26]. One of the most successful applications that the DSC algorithm has accomplished so far is the analysis of solid structures [24, 27-35]. The DSC algorithm was utilized to provide at least 11 significant digits for the first 100 modes of a simply supported square plate governed by the fourth-order biharmonic equations [27]. The DSC algorithm has been used for vibration analysis of plates with simply supported, clamped, and transversely supported edges [33], mixed edge supports [30], complex internal supports [24,32], irregular internal supports [35], and plates subjected to high-frequency vibration levels [31,34]. In particular, analysis of plates with irregular supports is a challenging task because of possible ill-conditioned matrix. Analysis of high-frequency vibration modes is another challenging problem in structural design [21]. Low-order methods, like $h$-version finite element methods, converge slowly for high-order modes. Standard global methods fail to work for high-order modes due to the numerical round off errors when the degree of polynomial is increased to a certain level. The DSC algorithm provides accurate prediction of thousands of vibration modes, which was not available to engineers previously [31,34].

Another distinct development in numerical analysis of plates is the $p b-2$ Rayleigh-Ritz method [36,37]. The method avoids the difficulty of global methods for implementing boundary conditions by appropriately choosing the basis function so that the boundary conditions are automatically satisfied. Essentially, the product of a two-dimensional polynomial function $(p-2)$ and a boundary function $(b)$ is utilized. The boundary function constitutes proper powers of polynomials that are simultaneous solutions to the differential equations of the boundary conditions. In the past decade, the $p b-2$ Rayleigh-Ritz method has had substantial success in the vibration analysis of Mindlin plates [37-43]. Much advance in this direction was summarized in a monograph [36].

The objective of the present work is to explore a new computational method, which is a combination of the DSC algorithm and $p b-2$ Rayleigh-Ritz method, and thus, called DSC-Ritz method. The essential idea is to utilize the local DSC approximations and the $p b-2$ RayleighRitz boundary functions. As a result, the DSC-Ritz method has the advantage of both methods. 
The method was proposed and tested on vibration analysis of beams and thin plates by two of the present authors [44]. This paper further investigates the efficiency and convergence of the DSC-Ritz method for the vibration analysis of Mindlin plates.

Albeit the proposed DSC-Ritz method has a distinct mathematical foundation, it shares some similarities with the smooth particle hydrodynamics [45,46], meshless method [47], and element-free $k p$-Ritz method $[48,49]$. The latter makes use of the cubic spline function to construct the shape function and the penalty method to enforce essential boundary conditions. Such similarities enhance our understanding of the philosophy of both the previous meshless type of methods and the DSC-Ritz.

The organization of this paper is as follows. Section 2 is devoted to theoretical formulations. The philosophy of the DSC algorithm is briefly discussed. The energy formulation of Mindlin plates is described. Detailed boundary conditions are given and the DSC-Ritz formalism is presented with two sets of new basis functions. In Section 3, the new method are applied to the numerical analysis of Mindlin plates with different shapes and combinations of boundary conditions. Validation is conducted by extensive convergence study and by a comparison with the literature. This paper ends with a conclusion.

\section{THEORETICAL FORMULATIONS}

In this section, a brief review is given to the theory of DSC before the formalism of Mindlin plates is described. The DSC-Ritz method of solution is introduced in the last subsection.

\subsection{Discrete singular convolution}

Singular convolutions occur commonly in many science and engineering problems and are a special class of mathematical transformations. It is most convenient to discuss singular convolutions in the context of the theory of distributions. The latter has a significant impact in mathematical analysis. It provides a rigorous justification for a number of informal manipulations in engineering and has significant influence over many mathematical disciplines, such as operator calculus, differential equations, functional analysis, harmonic analysis, harmonic analysis and transformation theory. Let $T$ be a distribution and $\eta(t)$ be an element of the space of test functions. A singular convolution can be defined as

$$
F(t)=(T \eta)(t)=\int_{-\infty}^{\infty} T(t-x) \eta(x) \mathrm{d} x
$$

Here $T(t-x)$ is a singular kernel. Depending on the form of the kernel $T$, the singular convolution is the key issue for a wide range of science and engineering problems, such as Hilbert transform, Abel transform and Radon transform. In the present study, only the singular kernels of the delta type are required

$$
T(x)=\delta^{(n)}(x), \quad(n=0,1,2, \ldots)
$$

Here, kernel $T(x)=\delta(x)$ is the delta distribution and is of particular importance for interpolation of surfaces and curves. Higher-order kernels, $T(x)=\delta^{(n)},(n=1,2, \ldots)$ are essential for numerically solving differential equations and for image processing, noise estimation, etc. However since these kernels are very singular, they cannot be directly digitized in computers. 
Hence, the singular convolution, (1), is of little direct numerical merit. To avoid the difficulty of using singular expressions directly in computer, we construct sequences of approximations $\left(T_{\alpha}\right)$ to the distribution $T$

$$
\lim _{\alpha \rightarrow \alpha_{0}} T_{\alpha}(x) \rightarrow T(x)
$$

where $\alpha_{0}$ is a generalized limit. Obviously, in the case of $T(x)=\delta(x)$, each element in the sequence, $T_{\alpha}(x)$, is a delta sequence kernel. With a sufficiently smooth approximation, it is useful to consider a discrete singular convolution

$$
F_{\alpha}(t)=\sum_{k} T_{\alpha}\left(t-x_{k}\right) f\left(x_{k}\right)
$$

where $F_{\alpha}(t)$ is an approximation to $F(t)$ and $\left\{x_{k}\right\}$ is an appropriate set of discrete points on which the DSC (4) is well defined. Note that, the original test function $\eta(x)$ has been replaced by $f(x)$.

Obviously, as the Fourier transform of the delta distribution is unit in the Fourier domain, the distribution can be regarded as a universal reproducing kernel [22]

$$
f(x)=\int \delta\left(x-x^{\prime}\right) f\left(x^{\prime}\right) \mathrm{d} x^{\prime}
$$

As a consequence, delta sequence kernels are approximate reproducing kernels or bandlimited reproducing kernels which provide good approximation to the universal reproducing kernel in certain frequency bands.

There are many delta sequence kernels arising in the theory of partial differential equations, Fourier transforms and signal analysis, with completely different mathematical properties. The reader is referred to References [22,24] for an elaboration on historical aspects of the delta distribution and its approximations. For the purpose of numerical computations, the delta sequence kernels of both positive type and Dirichlet type are of particular importance and have very distinct mathematical and numerical properties.

\section{Definition}

Let $\left\{\delta_{\alpha}\right\}$ be a sequence of kernel functions on $(-\infty, \infty)$ which are integrable over every bounded interval. We call $\left\{\delta_{\alpha}\right\}$ a delta sequence kernel of positive type if

1. $\int_{-a}^{a} \delta_{\alpha} \rightarrow 1$ as $\alpha \rightarrow \alpha_{0}$ for some finite constant $a$.

2. For every constant $\gamma>0,\left(\int_{-\infty}^{-\gamma}+\int_{\gamma}^{\infty}\right) \delta_{\alpha} \rightarrow 0$ as $\alpha \rightarrow \alpha_{0}$.

3. $\delta_{\alpha}(x) \geqslant 0$ for all $x$ and $\alpha$.

Although the delta sequence kernels of Dirichlet type have been extensively studied in our previous numerical work in the framework of the collocation, use of delta sequence kernels of positive type has rarely been considered. However, a variety of delta sequence kernels of positive type were described in detail in Reference [24]. Important examples include impulse functions, Gauss' delta sequence kernel, Lorentz's delta sequence kernel, Landau's delta sequence kernel, Poisson's delta sequence kernel family, Fejér's delta sequence kernel and its generalization. Most of these kernels were utilized in our previous test [44]. In this work, we 


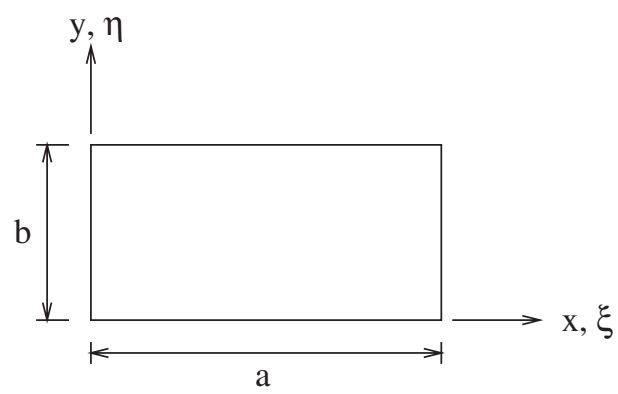

Figure 1. Geometry of rectangular Mindlin plate.

focus our study on two typical kernels, Gauss' delta sequence kernel

$$
\delta_{\sigma}(x)=\frac{1}{\sqrt{2 \pi} \sigma} \mathrm{e}^{-x^{2} / 2 \sigma^{2}}
$$

and Fejer's delta sequence kernel

$$
\delta_{k}(x)= \begin{cases}F_{k}(x) & |x| \leqslant \pi, \quad k=0,1,2 \ldots \\ 0 & \text { otherwise }\end{cases}
$$

where

$$
F_{k}(x)=\frac{\sin ^{2}\left(\frac{1}{2} k x\right)}{2 \pi k \sin ^{2}\left(\frac{1}{2} x\right)}, \quad-\infty<x<\infty
$$

A major advantage of many DSC delta sequence kernels is their localization. For example, the Gauss' kernel is an element of the Schwartz space functions. The decay property of the Fejer's kernel can be improved by choosing appropriate parameter $\sigma$. It was well understood that the use of delta sequence kernels of positive type has to be formulated in a Galerkin algorithm [23]. For conservative systems, such as vibration of plates, the Galerkin algorithm is essentially identical to the Ritz variational formulation. Thus, the rest of this section is devoted to these issues.

\subsection{Energy functionals of Mindlin plates}

In this subsection, we briefly review the theory of Mindlin plates to establish concepts and notations. More detailed description can be found elsewhere [40]. Let us consider a flat, isotropic, thick, rectangular (or triangular) plate of uniform thickness $h$, length $a$, width $b$, Young's modulus $E$, shear modulus $G$ and Poisson's ratio $v$. The geometry of a rectangular plate is shown in Figure 1. The plate may have an arbitrary combination of different supporting edges. The goal is to determine the natural frequencies of the plate.

According to the first-order shear deformable plate theory [4], the displacement fields of the plate in orthogonal co-ordinates can be expressed as

$$
u(x, y, z)=z \theta_{x}(x, y), \quad v(x, y, z)=z \theta_{y}(x, y), \quad w(x, y, z)=w(x, y)
$$


where $u, v$ are inplane displacements in the $x$-, and $y$-directions, respectively, $w$ the transverse displacement, and $\theta_{x}(x, y)$ and $\theta_{y}(x, y)$ the bending slopes along the $y$ - and $x$-axes, respectively. Note that $\theta_{x}$ and $\theta_{y}$ depend on variables $x$ and $y$, and the transverse displacement, $w$, is assumed to be independent of $z$, i.e. no thickness deformation is allowed. In view of Equation (9) and using Green's definition for strains,

$$
\boldsymbol{\varepsilon}=\left\{\begin{array}{llll}
\varepsilon_{x x} & \varepsilon_{y y}, \quad \gamma_{x y}, \quad \gamma_{x z}, \quad \gamma_{y z}
\end{array}\right\}^{\mathrm{T}}=\left\{\frac{\partial u}{\partial x}, \frac{\partial v}{\partial y}, \frac{\partial u}{\partial y}+\frac{\partial v}{\partial x}, \frac{\partial u}{\partial z}+\frac{\partial w}{\partial x}, \frac{\partial v}{\partial z}+\frac{\partial w}{\partial y}\right\}^{\mathrm{T}}
$$

where $\boldsymbol{\varepsilon}$ is the strain tensor having various non-vanishing components described in Equation (10).

To construct the energy functional, we consider the strain energy functional of the plate [40]

$$
U=\frac{1}{2} \int_{V} \varepsilon^{\mathrm{T}}[\mathbf{B}] \varepsilon \mathrm{d} V
$$

where $V$ is the volume of the plate, and $[\mathbf{B}]$ a matrix determined by material property and is given by

$$
[\mathbf{B}]=\left[\begin{array}{ccccc}
\frac{E}{1-v^{2}} & \frac{v E}{1-v^{2}} & 0 & 0 & 0 \\
\frac{v E}{1-v^{2}} & \frac{E}{1-v^{2}} & 0 & 0 & 0 \\
0 & 0 & G & 0 & 0 \\
0 & 0 & 0 & \kappa G & 0 \\
0 & 0 & 0 & 0 & \kappa G
\end{array}\right]
$$

where $G=E /[2(1+v)]$ and $\kappa$ is the shear correction factor that is used to compensate the error due to the assumption of constant transverse shear strain distribution over the plate thickness in the Mindlin plate theory.

The strain energy, Equation (11), can be evaluated from Equations (9), (10) and (12) to yield

$$
\begin{aligned}
U= & \frac{1}{2} \int_{V}\left\{\frac{E z^{2}}{1-v^{2}}\left[\left(\frac{\partial \theta_{x}}{\partial x}+\frac{\partial \theta_{y}}{\partial y}\right)^{2}-2(1-v)\left(\frac{\partial \theta_{x}}{\partial x} \frac{\partial \theta_{y}}{\partial y}-\frac{1}{4}\left(\frac{\partial \theta_{x}}{\partial y}+\frac{\partial \theta_{y}}{\partial x}\right)^{2}\right)\right]\right. \\
& \left.+\kappa G\left[\left(\theta_{x}+\frac{\partial w}{\partial x}\right)^{2}+\left(\theta_{y}+\frac{\partial w}{\partial y}\right)^{2}\right]\right\} \mathrm{d} V
\end{aligned}
$$

As expected, the well-known strain energy for thin plates can be obtained if we set $\theta_{x}=$ $-\partial w / \partial x$ and $\theta_{y}=-\partial w / \partial y$ in Equation (13).

Another important component in the energy functional is the kinetic energy $T$. For free vibration, the kinetic energy for the plate is given by $T=\frac{1}{2} \omega^{2} \int_{A}\left[\rho h w^{2}+\frac{1}{12} \rho h^{3}\left(\theta_{x}^{2}+\theta_{y}^{2}\right)\right] \mathrm{d} A$, where $\omega$ is the angular frequency and $\rho$ the mass density of the plate.

It is well-known that the total energy functional can be expressed as the difference of the strain and kinetic energy $\Pi=U-T$. The latter can be used as the starting point for numerical computations. 
Table I. Convergence study for rectangular plates with CCCC boundary conditions.

\begin{tabular}{|c|c|c|c|c|c|c|c|c|c|c|}
\hline \multirow[b]{2}{*}{$a / b$} & \multirow[b]{2}{*}{$h / b$} & \multirow[b]{2}{*}{ Kernel } & \multirow{2}{*}{$\begin{array}{l}\text { Parameter } \\
\text { of kernel }\end{array}$} & \multirow{2}{*}{$\begin{array}{l}\text { Number } \\
\text { of grid } \\
\text { points }\end{array}$} & \multicolumn{6}{|c|}{ Mode sequence number } \\
\hline & & & & & 1 & 2 & 3 & 4 & 5 & 6 \\
\hline \multirow[t]{26}{*}{1.0} & \multirow[t]{12}{*}{0.1} & \multirow[t]{6}{*}{ Gauss } & \multirow[t]{6}{*}{$r=1.8$} & $N=2$ & 5.1701 & 11.7620 & 11.7620 & 15.8121 & 67.0104 & 67.0104 \\
\hline & & & & $N=4$ & 3.3116 & 6.3073 & 6.3073 & 8.8231 & 13.2954 & 13.4748 \\
\hline & & & & $N=6$ & 3.2984 & 6.2891 & 6.2891 & 8.8145 & 10.3843 & 10.4813 \\
\hline & & & & $N=7$ & 3.2959 & 6.2884 & 6.2884 & 8.8169 & 10.3803 & 10.4789 \\
\hline & & & & $N=8$ & 3.2963 & 6.2868 & 6.2868 & 8.8113 & 10.3815 & 10.4800 \\
\hline & & & & $N=9$ & 3.2956 & 6.2867 & 6.2867 & 8.8122 & 10.3794 & 10.4783 \\
\hline & & \multirow[t]{6}{*}{ Fejer } & \multirow[t]{6}{*}{$k=2, r=3.0$} & $N=2$ & 5.0659 & 11.6873 & 11.6873 & 15.7348 & 67.0056 & 67.0056 \\
\hline & & & & $N=4$ & 3.3261 & 6.3049 & 6.3049 & 8.8273 & 12.7812 & 12.9637 \\
\hline & & & & $N=6$ & 3.2994 & 6.2896 & 6.2896 & 8.8148 & 10.3856 & 10.4824 \\
\hline & & & & $N=7$ & 3.2958 & 6.2881 & 6.2881 & 8.8163 & 10.3800 & 10.4788 \\
\hline & & & & $N=8$ & 3.2959 & 6.2864 & 6.2864 & 8.8105 & 10.3805 & 10.4790 \\
\hline & & & & $N=9$ & 3.2954 & 6.2861 & 6.2861 & 8.8109 & 10.3789 & 10.4780 \\
\hline & & Reference [50] & - & - & 3.2954 & 6.2858 & 6.2858 & 8.8098 & 10.3788 & 10.4778 \\
\hline & \multirow[t]{13}{*}{0.2} & \multirow[t]{6}{*}{ Gauss } & \multirow[t]{6}{*}{$r=1.8$} & $N=2$ & 3.0967 & 6.0297 & 6.0297 & 7.9581 & 17.7249 & 17.7249 \\
\hline & & & & $N=4$ & 2.6913 & 4.6967 & 4.6967 & 6.3031 & 7.8131 & 7.9568 \\
\hline & & & & $N=6$ & 2.6881 & 4.6913 & 4.6913 & 6.2991 & 7.1775 & 7.2765 \\
\hline & & & & $N=7$ & 2.6876 & 4.6911 & 4.6911 & 6.2994 & 7.1768 & 7.2761 \\
\hline & & & & $N=8$ & 2.6876 & 4.6909 & 4.6909 & 6.2987 & 7.1769 & 7.2762 \\
\hline & & & & $N=9$ & 2.6875 & 4.6908 & 4.6908 & 6.2988 & 7.1767 & 7.2760 \\
\hline & & \multirow[t]{6}{*}{ Fejer } & \multirow[t]{6}{*}{$k=2, r=3.0$} & $N=2$ & 3.0614 & 5.9964 & 5.9964 & 7.9215 & 17.7204 & 17.7204 \\
\hline & & & & $N=4$ & 2.6938 & 4.6945 & 4.6945 & 6.3016 & 7.6527 & 7.7922 \\
\hline & & & & $N=6$ & 2.6882 & 6.2991 & 7.1776 & 7.2766 & 10.0401 & 10.0401 \\
\hline & & & & $N=7$ & 2.6875 & 4.6910 & 4.6910 & 6.2993 & 7.1768 & 7.2761 \\
\hline & & & & $N=8$ & 2.6875 & 4.6908 & 4.6908 & 6.2986 & 7.1768 & 7.2761 \\
\hline & & & & $N=9$ & 2.6875 & 4.6908 & 4.6908 & 6.2986 & 7.1767 & 7.2760 \\
\hline & & Reference [50] & - & - & 2.6875 & 4.6907 & 4.6907 & 6.2985 & 7.1767 & 7.2759 \\
\hline \multirow[t]{19}{*}{2.0} & \multirow[t]{13}{*}{0.1} & \multirow[t]{6}{*}{ Gauss } & $r=1.8$ & $N=2$ & 3.9931 & 6.6569 & 11.2876 & 12.4816 & 66.2449 & 66.7871 \\
\hline & & & & $N=4$ & 2.3210 & 2.9639 & 5.5927 & 6.1438 & 6.3301 & 8.4179 \\
\hline & & & & $N=6$ & 2.3115 & 2.9546 & 4.0767 & 5.5732 & 5.6676 & 6.1296 \\
\hline & & & & $N=7$ & 2.3096 & 2.9541 & 4.0726 & 5.5727 & 5.6094 & 6.1317 \\
\hline & & & & $N=8$ & 2.3099 & 2.9525 & 4.0741 & 5.5716 & 5.6085 & 6.1270 \\
\hline & & & & $N=9$ & 2.3094 & 2.9525 & 4.0717 & 5.5714 & 5.6091 & 6.1277 \\
\hline & & Fejer & $k=2, r=3.0$ & $N=2$ & 3.9065 & 6.5928 & 11.2267 & 12.4198 & 66.2428 & 66.7837 \\
\hline & & & & $N=4$ & 2.3329 & 2.9726 & 5.5829 & 6.0194 & 6.1410 & 8.1921 \\
\hline & & & & $N=6$ & 2.3123 & 2.9551 & 4.0789 & 5.5736 & 5.6496 & 6.1301 \\
\hline & & & & $N=7$ & 2.3095 & 2.9539 & 4.0723 & 5.5725 & 5.6113 & 6.1312 \\
\hline & & & & $N=8$ & 2.3096 & 2.9521 & 4.0729 & 5.5712 & 5.6080 & 6.1264 \\
\hline & & & & $N=9$ & 2.3093 & 2.9519 & 4.0711 & 5.5709 & 5.6075 & 6.1265 \\
\hline & & Reference [50] & - & - & 2.3092 & 2.9515 & 4.0708 & 5.5708 & 5.6066 & 6.1256 \\
\hline & 0.2 & Gauss & $r=1.8$ & $N=2$ & 2.3537 & 3.5408 & 5.6688 & 6.2580 & 16.9896 & 17.5132 \\
\hline & & & & $N=4$ & 1.9529 & 2.4562 & 3.8709 & 4.2110 & 4.6042 & 5.5662 \\
\hline & & & & $N=6$ & 1.9501 & 2.4532 & 3.2918 & 4.2053 & 4.3958 & 4.5993 \\
\hline & & & & $N=7$ & 1.9496 & 2.4532 & 3.2906 & 4.2050 & 4.3717 & 4.5996 \\
\hline & & & & $N=8$ & 1.9496 & 2.4527 & 3.2909 & 4.2049 & 4.3710 & 4.5987 \\
\hline & & & & $N=9$ & 1.9495 & 2.4527 & 3.2903 & 4.2048 & 4.3711 & 4.5988 \\
\hline
\end{tabular}


Table I. Continued.

\begin{tabular}{|c|c|c|c|c|c|c|c|c|}
\hline \multirow{6}{*}{ Fejer } & $k=2, r=3.0$ & $N=2$ & 2.3226 & 3.5142 & 5.6394 & 6.2280 & 16.9876 & 17.5100 \\
\hline & & $N=4$ & 1.9555 & 2.4576 & 3.7638 & 4.2075 & 4.6030 & 5.4963 \\
\hline & & $N=6$ & 1.9502 & 2.4534 & 3.2922 & 4.2054 & 4.3917 & 4.5994 \\
\hline & & $N=7$ & 1.9496 & 2.4531 & 3.2905 & 4.2050 & 4.3727 & 4.5995 \\
\hline & & $N=8$ & 1.9496 & 2.4526 & 3.2906 & 4.2049 & 4.3708 & 4.5985 \\
\hline & & $N=9$ & 1.9495 & 2.4525 & 3.2902 & 4.2048 & 4.3708 & 4.5986 \\
\hline Reference [50] & - & - & 1.9495 & 2.4524 & 3.2901 & 4.2047 & 4.3706 & 4.5984 \\
\hline
\end{tabular}

Table II. Impact study of parameter $r$ on rectangular plates with SSSS boundary conditions with $a / b=1.0, h / b=0.1$ and $N=9$.

\begin{tabular}{|c|c|c|c|c|c|c|c|}
\hline \multirow[b]{2}{*}{ Kernel } & \multirow{2}{*}{$\begin{array}{l}\text { Parameter of } \\
\text { grid points }\end{array}$} & \multicolumn{6}{|c|}{ Mode sequence number } \\
\hline & & 1 & 2 & 3 & 4 & 5 & 6 \\
\hline \multirow[t]{11}{*}{ Gauss } & $r=1.0$ & 1.9453 & 4.6162 & 4.6162 & 7.0784 & 8.6202 & 8.6202 \\
\hline & $r=1.2$ & 1.9357 & 4.6106 & 4.6106 & 7.0736 & 8.6184 & 8.6184 \\
\hline & $r=1.4$ & 1.9326 & 4.6089 & 4.6089 & 7.0721 & 8.6182 & 8.6182 \\
\hline & $r=1.6$ & 1.9318 & 4.6085 & 4.6085 & 7.0718 & 8.6175 & 8.6175 \\
\hline & $r=1.8$ & 1.9317 & 4.6084 & 4.6084 & 7.0717 & 8.6167 & 8.6167 \\
\hline & $r=2.0$ & 1.9317 & 4.6084 & 4.6084 & 7.0717 & 8.6163 & 8.6163 \\
\hline & $r=2.2$ & 1.9317 & 4.6084 & 4.6084 & 7.0716 & 8.6162 & 8.6162 \\
\hline & $r=2.4$ & 1.9317 & 4.6084 & 4.6084 & 7.0716 & 8.6162 & 8.6162 \\
\hline & $r=2.6$ & 1.9317 & 4.6084 & 4.6084 & 7.0716 & 8.6162 & 8.6162 \\
\hline & $r=2.8$ & 1.9317 & 4.6084 & 4.6084 & 7.0716 & 8.6162 & 8.6162 \\
\hline & $r=3.0$ & 1.9317 & 4.6084 & 4.6084 & 7.0698 & 8.6162 & 8.6162 \\
\hline Fejer & $r=1.0$ & - & - & - & - & - & - \\
\hline \multirow[t]{10}{*}{$(k=2)$} & $r=1.2$ & - & - & - & - & - & - \\
\hline & $r=1.4$ & - & - & - & - & - & - \\
\hline & $r=1.6$ & - & - & - & - & - & - \\
\hline & $r=1.8$ & 2.3076 & 4.8234 & 4.8234 & 7.2601 & 8.9147 & 8.9195 \\
\hline & $r=2.0$ & 1.9736 & 4.6327 & 4.6327 & 7.0935 & 8.6279 & 8.6279 \\
\hline & $r=2.2$ & 1.9357 & 4.6110 & 4.6110 & 7.0741 & 8.6168 & 8.6168 \\
\hline & $r=2.4$ & 1.9321 & 4.6086 & 4.6086 & 7.0719 & 8.6171 & 8.6171 \\
\hline & $r=2.6$ & 1.9317 & 4.6084 & 4.6084 & 7.0717 & 8.6166 & 8.6166 \\
\hline & $r=2.8$ & 1.9317 & 4.6084 & 4.6084 & 7.0716 & 8.6163 & 8.6163 \\
\hline & $r=3.0$ & 1.9317 & 4.6084 & 4.6084 & 7.0716 & 8.6162 & 8.6162 \\
\hline Reference [50] & - & 1.9317 & 4.6084 & 4.6084 & 7.0716 & 8.6162 & 8.6162 \\
\hline
\end{tabular}

With normalized co-ordinates $\xi=x / a, \eta=y / b$, the integration of Equation (13) with respect to $z$ yields

$$
\begin{aligned}
U= & \frac{1}{2} \int_{\bar{A}}\left\{D\left[\left(\frac{1}{a} \frac{\partial \theta_{x}}{\partial \xi}+\frac{1}{b} \frac{\partial \theta_{y}}{\partial \eta}\right)^{2}-2(1-v)\left(\frac{1}{a b} \frac{\partial \theta_{x}}{\partial \xi} \frac{\partial \theta_{y}}{\partial \eta}-\frac{1}{4}\left(\frac{1}{b} \frac{\partial \theta_{x}}{\partial \eta}+\frac{1}{a} \frac{\partial \theta_{y}}{\partial \xi}\right)^{2}\right)\right]\right. \\
& \left.+\kappa G h\left[\left(\theta_{x}+\frac{1}{a} \frac{\partial w}{\partial \xi}\right)^{2}+\left(\theta_{y}+\frac{1}{b} \frac{\partial w}{\partial \eta}\right)^{2}\right]\right\} a b \mathrm{~d} \bar{A}
\end{aligned}
$$

Copyright (C) 2004 John Wiley \& Sons, Ltd.

Int. J. Numer. Meth. Engng 2005; 62:262-288 
Table III. Impact study of parameter $k$ on rectangular plates with SSSS boundary conditions with $a / b=1.0, h / b=0.1$ and $N=9$.

\begin{tabular}{lccccccc}
\hline & & \multicolumn{6}{c}{ Mode sequence number } \\
\cline { 3 - 8 } Kernel & $\begin{array}{c}\text { Parameter of } \\
\text { grid points }\end{array}$ & 1 & 2 & 3 & 4 & 5 & 6 \\
\hline Fejer & $k=1$ & - & - & - & - & - & - \\
$(r=3.0)$ & $k=2$ & 1.9317 & 4.6084 & 4.6084 & 7.0716 & 8.6162 & 8.6162 \\
& $k=3$ & 1.9317 & 4.6084 & 4.6084 & 7.0717 & 8.6163 & 8.6163 \\
& $k=4$ & 1.9317 & 4.6084 & 4.6084 & 7.0717 & 8.6164 & 8.6164 \\
& $k=5$ & 1.9318 & - & - & 7.0717 & 8.6164 & 8.6164 \\
& $k=6$ & 1.9318 & 4.6084 & 4.6084 & 7.0717 & 8.6164 & 8.6164 \\
& $k=7$ & 1.9318 & 4.6084 & 4.6084 & 7.0717 & 8.6164 & 8.6164 \\
& $k=8$ & 1.9318 & 4.6084 & 4.6084 & 7.0717 & 8.6164 & 8.6164 \\
Reference [50] & $k=9$ & 1.9318 & 4.6084 & 4.6084 & 7.0717 & 8.6164 & 8.6164 \\
& $k=10$ & 1.9318 & 4.6084 & 4.6084 & 7.0717 & 8.6164 & 8.6164 \\
& - & 1.9317 & 4.6084 & 4.6084 & 7.0716 & 8.6162 & 8.6162 \\
\hline
\end{tabular}

and

$$
T=\frac{1}{2} \omega^{2} \int_{\bar{A}}\left[\rho h w^{2}+\frac{1}{12} \rho h^{3}\left(\theta_{x}^{2}+\theta_{y}^{2}\right)\right] a b \mathrm{~d} \bar{A}
$$

where $D=E h^{3} /\left[12\left(1-v^{2}\right)\right]$, and $\bar{A}$ is the non-dimensionalized area of the plate and $\mathrm{d} \bar{A}=\mathrm{d} \xi \mathrm{d} \eta$.

\subsection{Boundary conditions}

The most commonly occurring support conditions for Mindlin plates are [36]:

(a) Free edge $(\mathrm{F})$ - Boundary conditions for free edges are given by

$$
Q_{n}=0, \quad M_{n}=0 \quad \text { and } \quad M_{n t}=0
$$

where $Q_{n}$ is the shearing force, $M_{n}$ the bending moment and $M_{n t}$ the twisting moment.

(b) Simply supported edge (S)-Simply supported edges for rectangular Mindlin plates are given by

$$
w=0, \quad M_{n}=0 \quad \text { and } \quad \theta_{t}=0
$$

where $\theta_{t}$ is the rotation of the mid-plane normal in the tangent plane to the plate boundary.

(c) Simply supported edge $\left(\mathrm{S}^{*}\right)$-There is another kind (second kind) of simply supported boundary conditions, which is used for triangular Mindlin plates. The $\mathrm{S}^{*}$ conditions state that

$$
w=0, \quad M_{n}=0 \quad \text { and } \quad M_{n t}=0
$$

(d) Clamped edge (C)-Clamped edges are constrained by

$$
w=0, \quad \theta_{n}=0 \quad \text { and } \quad \theta_{t}=0
$$

where $\theta_{n}$ is the rotation of the mid-plane normal to the clamped edge. 
Table IV. Comparison study for rectangular plates with CSSF boundary conditions.

\begin{tabular}{|c|c|c|c|c|c|c|c|c|c|}
\hline \multirow[b]{2}{*}{$a / b$} & \multirow[b]{2}{*}{$h / b$} & \multirow[b]{2}{*}{ Kernel } & \multirow{2}{*}{$\begin{array}{c}\text { Parameter of } \\
\text { kernel }\end{array}$} & \multicolumn{6}{|c|}{ Mode sequence number } \\
\hline & & & & 1 & 2 & 3 & 4 & 5 & 6 \\
\hline \multirow[t]{6}{*}{0.6} & \multirow[t]{3}{*}{0.1} & Gauss & $r=2.2$ & 3.9925 & 5.1760 & 7.7095 & 11.0451 & 11.5248 & 12.1483 \\
\hline & & Fejer & $=2, r=3.0$ & 3.9921 & 5.1750 & 7.7079 & 11.0439 & 5232 & \\
\hline & & Reference [50] & - & 3.9914 & 5.1735 & 7.7053 & 11.0416 & 11.5201 & 12.1436 \\
\hline & \multirow[t]{3}{*}{0.2} & Gauss & $r=2.2$ & 3.1819 & 4.0443 & 5.8257 & 7.6466 & 8.2754 & $8.35^{\circ}$ \\
\hline & & Fejer & $=2, r=3.0$ & 3.1818 & 4.0441 & 5.8254 & 7.6463 & & 571 \\
\hline & & Reference [50] & - & 3.1817 & 4.0438 & 5.8250 & 7.6458 & 8.2745 & 8.3564 \\
\hline \multirow[t]{6}{*}{1.0} & \multirow[t]{3}{*}{0.1} & Gauss & $r=2$ & 1. & 2.9170 & 4.6 & 5.76 & 3 & 8.57 \\
\hline & & Fejer & $k=2, r=$ & 1.6198 & 2.9175 & 4.6621 & 5.7689 & & 779 \\
\hline & & Reference [50] & - & 1.6195 & 2.9165 & 4.6612 & 5.7675 & 5.9711 & 8.5744 \\
\hline & \multirow{3}{*}{0.2} & Gauss & $r=2.2$ & 1.4452 & 2.4997 & 3.7407 & 4.6356 & 4.6770 & 6.4321 \\
\hline & & Fejer & $k=2, r=3.0$ & 1.4452 & 2.4997 & 3.7408 & 4.6358 & 4.6771 & 6.4328 \\
\hline & & Reference [50] & - & 1.4451 & 2.4996 & 3.7406 & 4.6355 & 4.6769 & 6.4323 \\
\hline \multirow[t]{6}{*}{1.5} & \multirow[t]{3}{*}{0.1} & Gauss & $r=2.2$ & 0.8085 & 2.1511 & 2.2748 & 3.6625 & 4.3984 & 5.1505 \\
\hline & & Fejer & $=2, r=3.0$ & 0.8083 & 2.1507 & 2.2743 & 3.6616 & 6 & 0 \\
\hline & & Reference [50] & - & 0.8081 & 2.1500 & 2.2739 & 3.6601 & 4.3968 & 5.1492 \\
\hline & \multirow[t]{3}{*}{0.2} & Gauss & $r=2.2$ & 0.7576 & 1.9207 & 1.9980 & 3.0821 & 36 & 424 \\
\hline & & Fejer & $=2, r=$ & 0.7575 & 1.9206 & 1.9979 & 3.0819 & 20 & 4.2405 \\
\hline & & Reference [50] & - & 0.7575 & 1.9205 & 1.9978 & 3.0817 & 3.6018 & 4.2404 \\
\hline \multirow[t]{6}{*}{2.0} & \multirow[t]{3}{*}{0.1} & Gauss & $r=2.2$ & 0.5101 & 1.3735 & & & & \\
\hline & & Fejer & $=2, r=3.0$ & 0.5101 & 1.3737 & 1.8747 & 2.6386 & 2.7766 & 4.0652 \\
\hline & & Reference [50] & - & 0.5100 & 1.3734 & 1.8742 & 2.6380 & 2.7754 & 4.0633 \\
\hline & \multirow[t]{3}{*}{0.2} & Gauss & $r=2.2$ & 0.4873 & 1.2599 & 1.7036 & 2.3007 & 0 & 33 \\
\hline & & & $=2, r=$ & 0.4873 & 1.2599 & 1.7 & 08 & & 3 \\
\hline & & Reference [50] & - & 0.4872 & 1.2598 & 1.7035 & 2.3006 & 2.4199 & 3.39 \\
\hline \multirow[t]{6}{*}{2.5} & \multirow[t]{3}{*}{0.1} & Gau & $1-2$ & 0.3659 & 0.9417 & 1.7 & 1. & & 2. \\
\hline & & Feje & $=2, r=$ & 0.3658 & 0.9416 & 1.7461 & 1.7789 & 2.3 & 2.8 \\
\hline & & Reference [50] & - & 0.3657 & 0.9413 & 1.7457 & 1.7785 & 2.3437 & 2.8686 \\
\hline & \multirow[t]{3}{*}{0.2} & Gaus & $r=2.2$ & 0.3526 & 0.8833 & 1.6 & 92 & 40 & 15 \\
\hline & & Fejer & $k=2, r=3.0$ & 0.3526 & 0.8834 & 1.6007 & 1.6091 & 2.0840 & 2.4912 \\
\hline & & Reference [50] & - & 0.3526 & 0.8833 & 1.6006 & 1.6090 & 2.0837 & 2.4909 \\
\hline
\end{tabular}

\subsection{The DSC-Ritz method}

In the DSC-Ritz method, we approximate the transverse deflection and the bending slopes by

$$
w(\xi, \eta)=\sum_{i=1}^{N} \sum_{j=1}^{N} c_{m} \phi_{m}(\xi, \eta), \theta_{x}(\xi, \eta)=\sum_{i=1}^{N} \sum_{j=1}^{N} d_{m} \psi_{x m}(\xi, \eta), \theta_{y}(\xi, \eta)=\sum_{i=1}^{N} \sum_{j=1}^{N} e_{m} \psi_{y m}(\xi, \eta)
$$


Table V. Comparison study for rectangular plates with CFSF boundary conditions.

\begin{tabular}{|c|c|c|c|c|c|c|c|c|c|}
\hline \multirow[b]{2}{*}{$a / b$} & \multirow[b]{2}{*}{$h / b$} & \multirow[b]{2}{*}{ Kernel } & \multirow{2}{*}{$\begin{array}{c}\text { Parameter of } \\
\text { kernel }\end{array}$} & \multicolumn{6}{|c|}{ Mode sequence number } \\
\hline & & & & 1 & 2 & 3 & 4 & 5 & 6 \\
\hline \multirow[t]{6}{*}{0.6} & \multirow[t]{3}{*}{0.1} & Gauss & $r=2.2$ & 3.8551 & 4.3259 & 5.9149 & 8.7782 & 10.9140 & 11.3617 \\
\hline & & Fejer & $=2, r=3.0$ & 3.8546 & 4.3255 & 5.9122 & 8.7774 & 10.9129 & 11.3610 \\
\hline & & Reference [50] & - & 3.8539 & 4.3185 & 5.9067 & 8.7540 & 10.9102 & 11.3454 \\
\hline & \multirow[t]{3}{*}{0.2} & Gauss & $r=2.2$ & 3.0817 & 3.4128 & 4.5432 & 6.4929 & 7.5612 & 7.8452 \\
\hline & & Fejer & $=2, r=$ & 3.0816 & 3.4126 & 4.5424 & 6.4926 & 7.5614 & 8450 \\
\hline & & Reference [50] & - & 3.0815 & 3.4108 & 4.5416 & 6.4876 & 7.5603 & 7.8406 \\
\hline \multirow[t]{6}{*}{1.0} & \multirow[t]{3}{*}{0.1} & Gauss & $r=2$. & 4738 & 1.9536 & 3.6499 & 4.5031 & .04 & 6.7 \\
\hline & & Fejer & $k=2, r=$ & 1.4737 & 1.9536 & 3.6483 & 4.5026 & 5.0479 & 6.7874 \\
\hline & & Reference [50] & - & 1.4735 & 1.9491 & 3.6452 & 4.5017 & 5.0395 & 6.7807 \\
\hline & \multirow[t]{3}{*}{0.2} & Gauss & $r=2.2$ & 1.3255 & 1.7030 & 3.0534 & 3.6265 & 054 & 5.2086 \\
\hline & & Fejer & $k=2, r=3.0$ & 1.3254 & 1.7030 & 3.0530 & 3.6264 & .0052 & 5.2075 \\
\hline & & Reference [50] & - & 1.3254 & 1.7019 & 3.0526 & 3.6262 & 4.0031 & 5.2064 \\
\hline \multirow[t]{6}{*}{1.5} & \multirow[t]{3}{*}{0.1} & Gauss & $r=2.2$ & 0.6666 & 1.1014 & 2.1049 & 2.6693 & 2.8575 & 4.2273 \\
\hline & & Fejer & $k=2, r=3.0$ & 0.6665 & 1.1015 & 2.1047 & 2.6691 & 5 & 269 \\
\hline & & Reference [50] & - & 0.6664 & 1.0978 & 2.1044 & 2.6635 & 2.8547 & 4.2261 \\
\hline & \multirow[t]{3}{*}{0.2} & Gauss & $r=2.2$ & 0.6320 & 1.0008 & 1.8622 & 2.2975 & 2.4 & 3.4 \\
\hline & & Fejer & $=2, r=3.0$ & 0.6320 & 1.0008 & 1.8622 & 2.2974 & 22 & 3.4778 \\
\hline & & Reference [50] & & 0.6320 & 0.9999 & 1.8621 & 2.2960 & 2.4920 & 3.4776 \\
\hline \multirow[t]{6}{*}{2.0} & \multirow[t]{3}{*}{0.1} & Gauss & $r=2.2$ & 0.3766 & 0.7610 & 1.2045 & & 6 & 2.5 \\
\hline & & Fejer & $=2, r=$ & 0.3766 & 0.7612 & 1.2043 & 1.7474 & 2.4604 & 2.5660 \\
\hline & & Reference [50] & - & 0.3765 & 0.7578 & 1.2041 & 1.7428 & 2.4599 & 2.5648 \\
\hline & \multirow[t]{3}{*}{0.2} & Gauss & $r=2.2$ & 0.3649 & 0.7025 & & 14 & & 2.2 \\
\hline & & Feje & $=2, r=3.0$ & 0.3648 & & & 1. & & 6 \\
\hline & & Reference [50] & 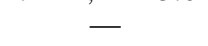 & 0.3648 & 0.7016 & 1.1152 & 1.5601 & 2.1600 & 2.2804 \\
\hline \multirow[t]{6}{*}{2.5} & \multirow[t]{3}{*}{0.1} & Gauss & $r=2.2$ & 0.2413 & 0.5817 & 0.7761 & 1.2859 & & 2.1852 \\
\hline & & Fej & $=2, r=$ & 0.2412 & 0.5820 & 0.7760 & 1.2859 & & 2.1850 \\
\hline & & Reference [50] & - & 0.2412 & 0.5785 & 0.7759 & 1.2818 & 1.5991 & 2.1798 \\
\hline & \multirow[t]{3}{*}{0.2} & Gaus & $r=2$ & 0.2362 & 0.5409 & 0.7 & 1.1714 & 1. & 1.9 \\
\hline & & Fei & $2, r=$ & 0.2362 & 0.5410 & 0.7365 & 1.1713 & 1.4 & 1.9296 \\
\hline & & Reference [50] & - & 0.2362 & 0.5400 & 0.7365 & 1.1702 & 1.4592 & 1.9281 \\
\hline
\end{tabular}

where $N$ is the number of grid points in both $x$ - and $y$-direction, $c_{m}, d_{m}$ and $e_{m}$ are the unknown coefficients, and the subscript $m$ is determined by

$$
m=(i-1) N+j
$$

and

$$
\phi_{m}=\delta_{\sigma i j}(\xi, \eta) B_{w}, \quad \psi_{x m}=\delta_{\sigma i j}(\xi, \eta) B_{x}, \quad \psi_{y m}=\delta_{\sigma i j}(\xi, \eta) B_{y}
$$



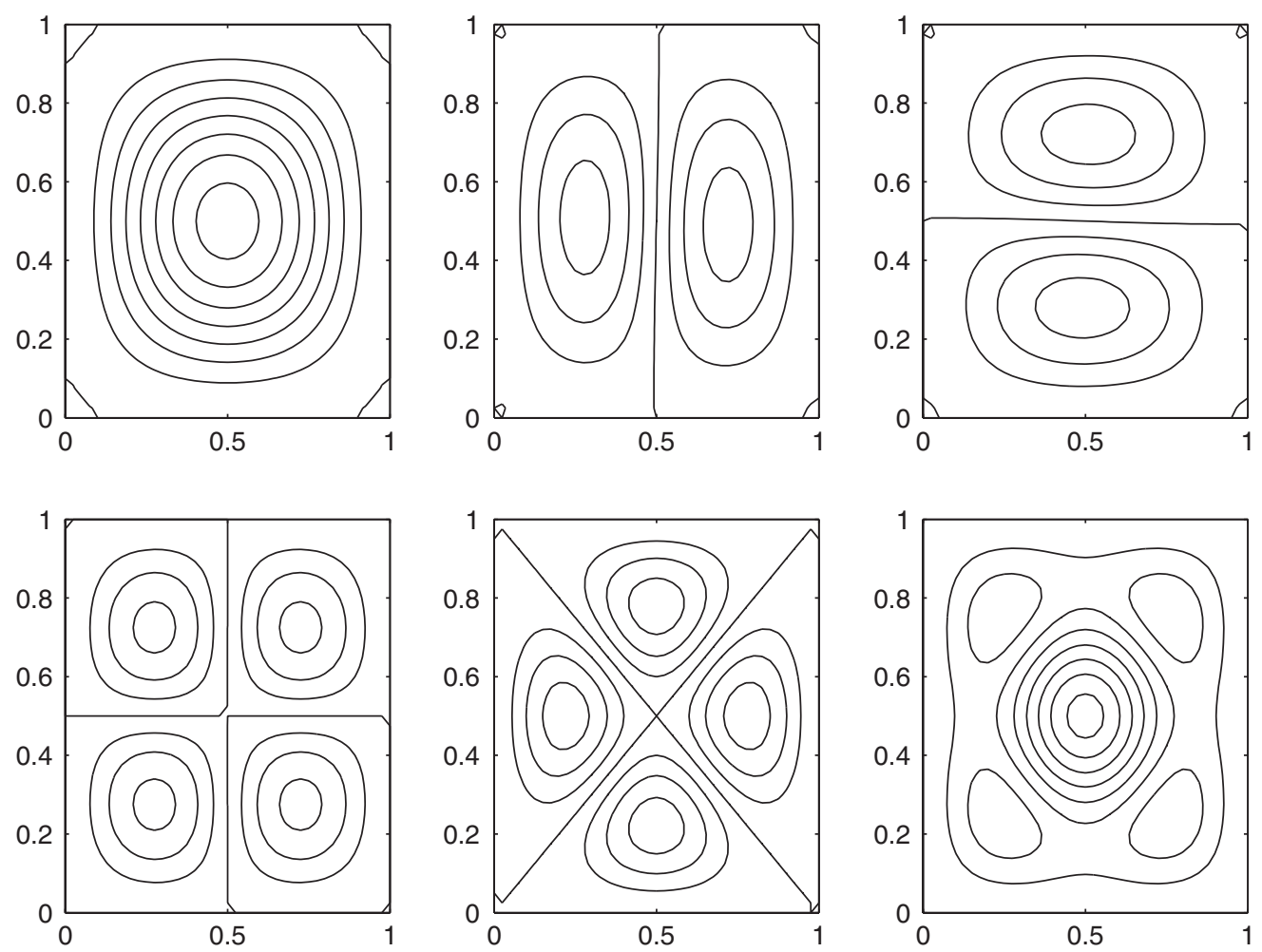

Figure 2. First six mode shapes of a CCCC rectangular plate $(a / b=1.0, h / b=0.10)$.

where $B_{w}, B_{x}$ and $B_{y}$ are boundary functions. We adopt the idea from the $p b-2$ Ritz method for the treatment of boundary conditions. Namely, the boundary functions must satisfy the geometric boundary conditions given by expressions in Equations (15)-(18). Therefore, boundary functions for the deflection can be expressed as [40]

$$
B_{w}=\prod_{j=1}^{L}\left[\Gamma_{j}(\xi, \eta)\right]^{\Omega_{j}}
$$

where $\Gamma_{j}$ is the boundary equation of the $j$ th supporting edge, $L$ is the number of plate edges (for rectangle $L=4$, for triangle $L=3$ ), and $\Omega_{j}$ depending on the support edge condition, takes the form of

$$
\begin{aligned}
& \Omega_{j}=0 \text { if the } j \text { th edge is free }(\mathrm{F}) \\
& \Omega_{j}=1 \text { if the } j \text { th edge is clamped (C) or simply supported ( } \mathrm{S} \text { and } \mathrm{S}^{*} \text { ) }
\end{aligned}
$$



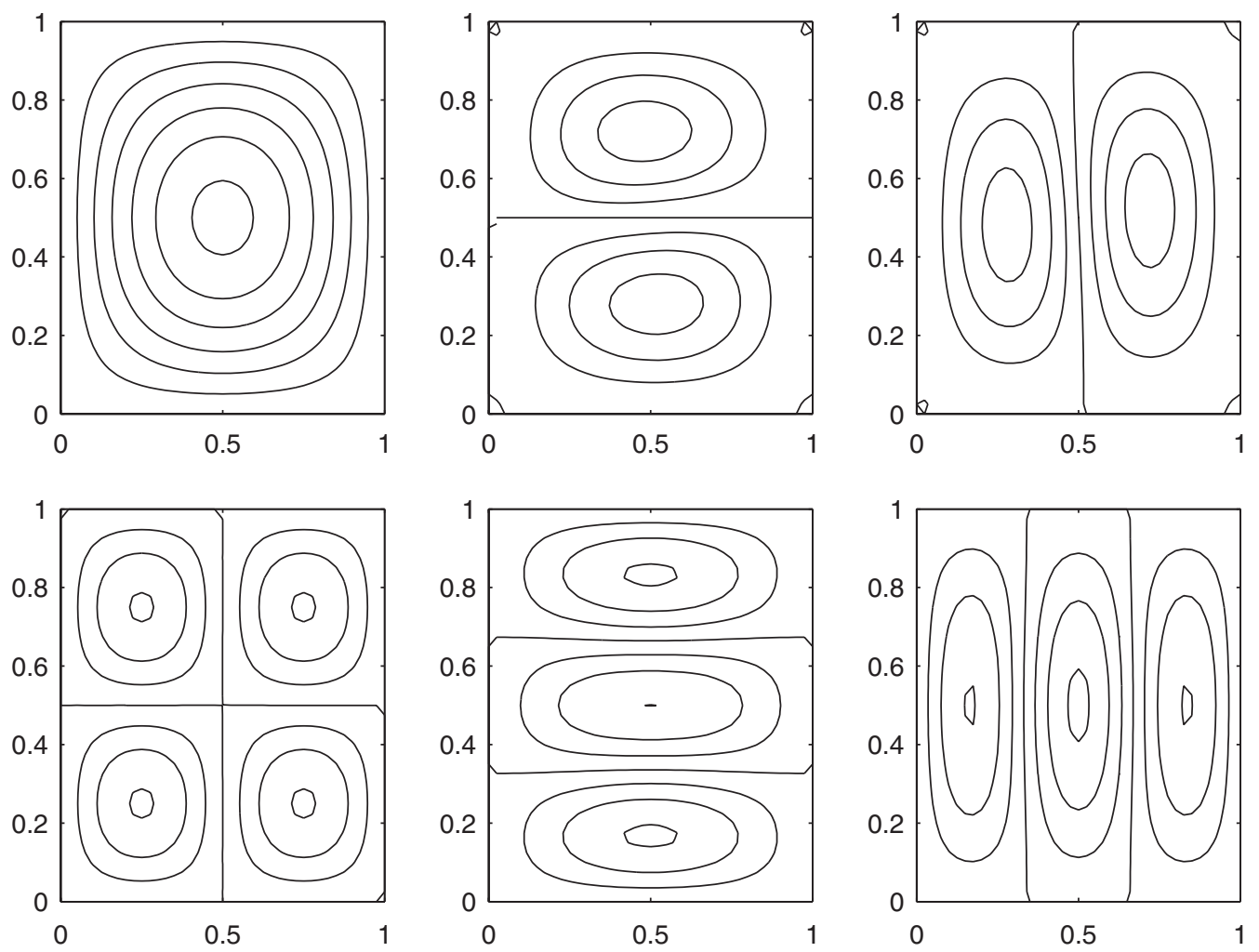

Figure 3. First six mode shapes of an SSSS rectangular plate $(a / b=1.0, h / b=0.10)$.

The boundary functions for the bending slopes can be expressed as

$$
B_{x}=\prod_{j=1}^{L}\left[\Gamma_{j}(\xi, \eta)\right]^{\Omega_{j}}
$$

$\Omega_{j}=0$ if the $j$ th edge is free (F) or simply supported $\left(\mathrm{S}^{*}\right)$ or $(\mathrm{S})$ in the $y$-direction

$\Omega_{j}=1$ if the $j$ th edge is clamped (C) or simply supported (S) in the $x$-direction

and

$$
B_{y}=\prod_{j=1}^{L}\left[\Gamma_{j}(\xi, \eta)\right]^{\Omega_{j}}
$$

$\Omega_{j}=0$ if the $j$ th edge is free (F) or simply supported $\left(\mathrm{S}^{*}\right)$ or $(\mathrm{S})$ in the $x$-direction 

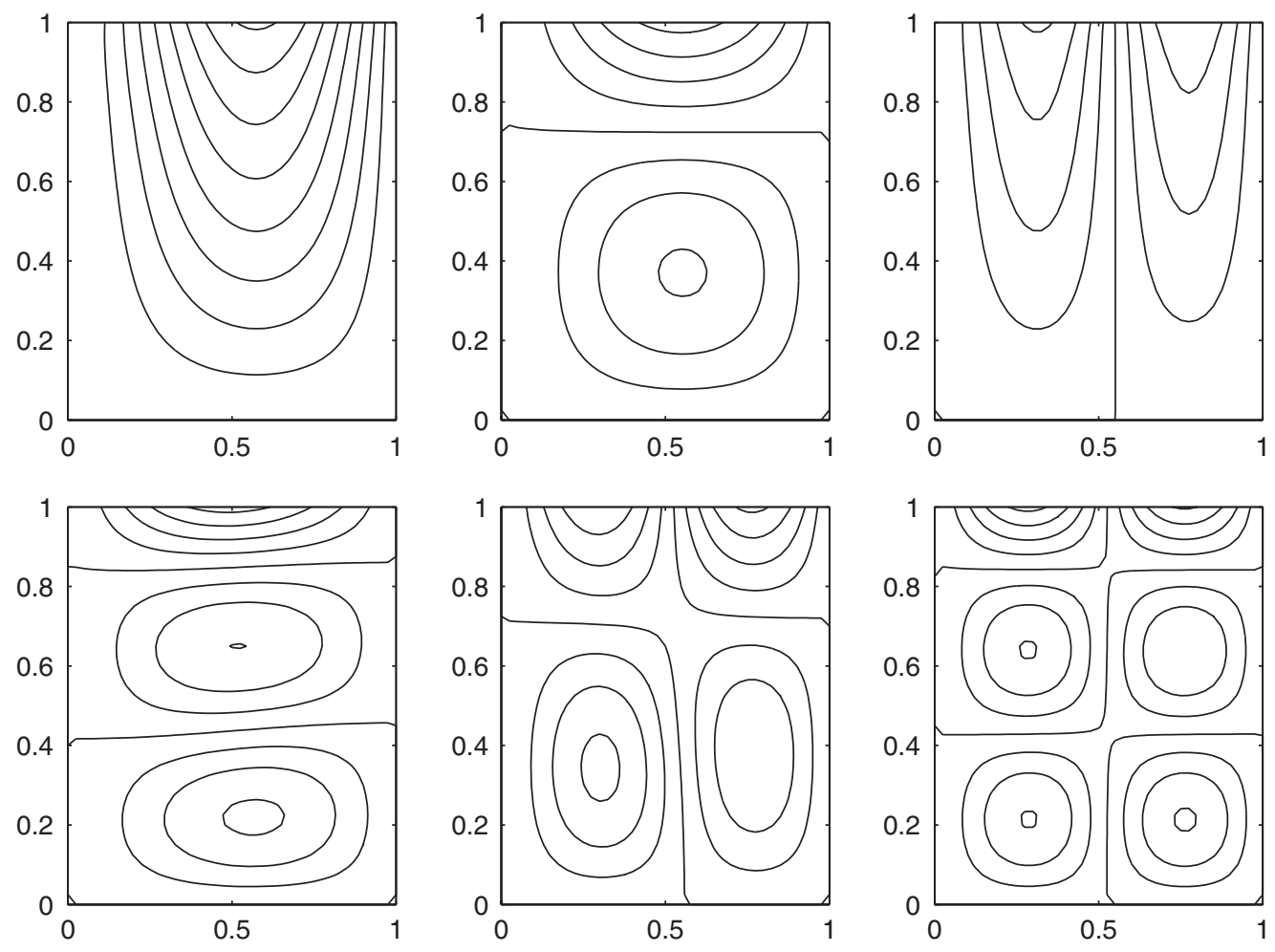

Figure 4. First six mode shapes of a CSSF rectangular plate $(a / b=1.0, h / b=0.10)$.

$\Omega_{j}=1$ if the $j$ th edge is clamped (C) or simply supported (S) in the $y$-direction

Note that $\delta_{\sigma i j}(\xi, \eta)$ in Equation (21) is a DSC delta kernel. Up to an arbitrary constant which is taken care by the minimization, the two-dimensional forms for the aforementioned two DSC kernels can be given by

$$
\delta_{\sigma i j}(x, y)=\mathrm{e}^{-\left(x-x_{i}\right)^{2} / 2 \sigma^{2}} \mathrm{e}^{-\left(y-y_{j}\right)^{2} / 2 \sigma^{2}}
$$

for Gauss' kernel, and

$$
\delta_{\sigma i j}(x, y)=\frac{\sin ^{2}\left(\frac{\pi}{\sigma}\left(x-x_{i}\right)\right) \sin ^{2}\left(\frac{\pi}{\sigma}\left(y-y_{j}\right)\right)}{\sin ^{2}\left(\frac{\pi}{\sigma}\left(x-x_{i}\right) /(2 k+1)\right) \sin ^{2}\left(\frac{\pi}{\sigma}\left(y-y_{j}\right) /(2 k+1)\right)}, \quad k=1,2, \ldots
$$

for Fejer's kernel. In all the above kernels, $x_{i}$ and $y_{j}$ are grid point co-ordinates along the $x$ and $y$-axis, respectively. The parameter $\sigma$ are chosen as $\sigma=r \Delta$, where $\Delta$ is the grid spacing, and $r$ is an adjustable parameter determining the radius of influence, and is usually chosen between 1.0 and 3.0 in this work. 

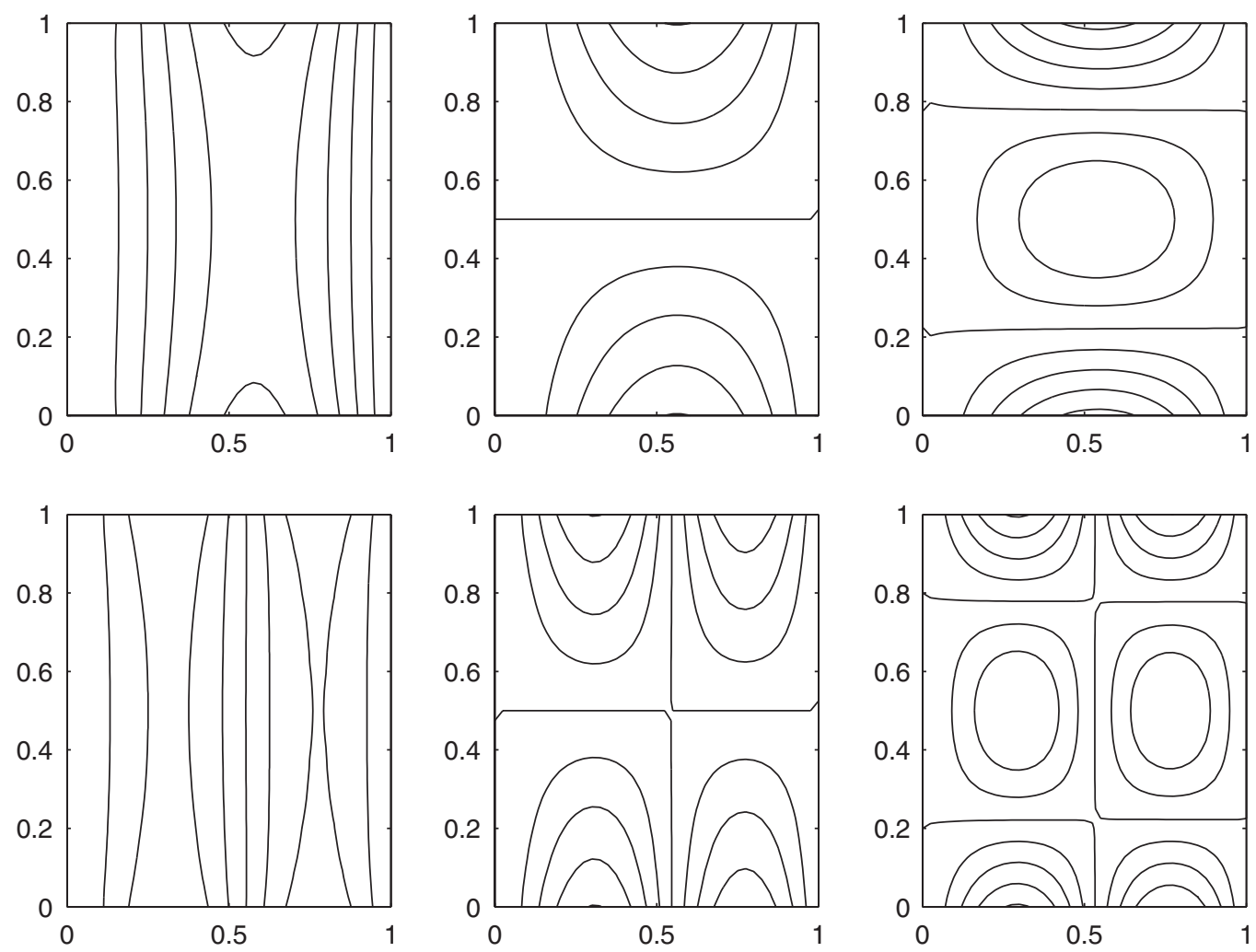

Figure 5. First six mode shapes of a CFSF rectangular plate $(a / b=1.0, h / b=0.10)$.

To determine the unknown coefficients, we substitute the Ritz trial functions in Equation (19) into the total energy functional $\Pi=U-T$ and minimize the latter with respect to the unknown coefficients. As a result, we obtain a set of linear algebraic equations

$$
\left([\mathbf{K}]-\omega^{2}[\mathbf{M}]\right)\left\{\begin{array}{l}
\{\mathbf{c}\} \\
\{\mathbf{d}\} \\
\{\mathbf{e}\}
\end{array}\right\}=\{\mathbf{0}\}
$$

where vectors $\mathbf{c}, \mathbf{d}$ and $\mathbf{e}$ are given by

$$
\{\mathbf{c}\}=\left\{\begin{array}{c}
c_{1} \\
c_{2} \\
\vdots \\
c_{p}
\end{array}\right\} ; \quad\{\mathbf{d}\}=\left\{\begin{array}{c}
d_{1} \\
d_{2} \\
\vdots \\
d_{q}
\end{array}\right\} ; \quad\{\mathbf{e}\}=\left\{\begin{array}{c}
e_{1} \\
e_{2} \\
\vdots \\
e_{r}
\end{array}\right\}
$$


Table VI. Convergence study for triangular plates with CCC boundary conditions, $h / b=0.15$.

\begin{tabular}{|c|c|c|c|c|c|c|c|c|}
\hline \multirow[b]{2}{*}{$\beta^{\circ}$} & \multirow[b]{2}{*}{ Kernel } & \multirow{2}{*}{$\begin{array}{l}\text { Number of } \\
\text { grid points }\end{array}$} & \multicolumn{6}{|c|}{ Mode sequence number } \\
\hline & & & 1 & 2 & 3 & 4 & 5 & 6 \\
\hline \multirow[t]{9}{*}{$30^{\circ}$} & Gauss & $N=7$ & 6.4541 & 9.4010 & 12.1168 & 12.4831 & 15.9068 & 16.0461 \\
\hline & $(r=2.2)$ & & 6.4540 & 9.4005 & 12.1159 & 12.4624 & 15.6491 & 15.9005 \\
\hline & & $N=9$ & 6.4538 & 9.4004 & 12.1155 & 12.4606 & 15.6164 & 15.8997 \\
\hline & & $N=10$ & 6.4537 & 9.4004 & 12.1152 & 12.4606 & 15.6133 & 15.8994 \\
\hline & Fejer & $N=6$ & 6.4550 & 9.4189 & 12.1201 & 12.7464 & 15.9591 & 17.7321 \\
\hline & $(r=3.0)$ & $N=7$ & 6.4545 & 9.4046 & 12.1183 & 12.5210 & 15.9135 & 16.0755 \\
\hline & & $N=8$ & 6.4544 & 9.4017 & 12.1171 & 12.4734 & 15.7607 & 15.9050 \\
\hline & & $N=9$ & 6.4543 & 9.4016 & 12.1167 & 12.4631 & 15.6429 & 15.9021 \\
\hline & Reference [40] & - & 6.454 & 9.402 & 12.12 & 12.46 & 15.64 & 15.90 \\
\hline \multirow[t]{9}{*}{$60^{\circ}$} & Gaus & $N=7$ & 10.2784 & 16.6672 & 16.6677 & 23.1279 & 23.9966 & 23.9975 \\
\hline & $(r=2.2)$ & $N=8$ & 10.2782 & 16.6671 & 16.6672 & 23.1275 & 23.9960 & 23.9966 \\
\hline & & $N=9$ & 10.2782 & 16.6669 & 16.6671 & 23.1273 & 23.9959 & 23.9963 \\
\hline & & $N=10$ & 10.2781 & 16.6669 & 16.6670 & 23.1272 & 23.9958 & 23.9961 \\
\hline & Fejer & $N=6$ & 10.2796 & 16.6704 & 16.6706 & 23.1634 & 24.0138 & 24.0473 \\
\hline & $(r=3.0)$ & $N=7$ & 10.2794 & 16.6697 & 16.6698 & 23.1322 & 24.0017 & 24.0034 \\
\hline & & $N=8$ & 10.2794 & 16.6696 & 16.6697 & 23.1316 & 24.0004 & 24.0004 \\
\hline & & $N=9$ & 10.2794 & 16.6696 & 16.6696 & 23.1315 & 24.0003 & 24.0003 \\
\hline & Reference [40] & - & 10.28 & 16.67 & 16.67 & 23.13 & 24.00 & 24.00 \\
\hline \multirow[t]{9}{*}{$90^{\circ}$} & Gauss & $N=7$ & 15.9415 & 22.9157 & 26.5298 & 30.8848 & 33.9444 & 38.4088 \\
\hline & $(r=1.2)$ & & 15.9410 & 22.9155 & 26.5294 & 30.8834 & 33.8866 & 38.3859 \\
\hline & & $N-9$ & 15.9411 & 22.9155 & 26.5288 & 30.8830 & 33.8857 & 38.3723 \\
\hline & & $N=10$ & 15.9409 & 22.9155 & 26.5289 & 30.8830 & 33.8854 & 38.3717 \\
\hline & Fejer & $N=6$ & 15.9446 & 22.9264 & 26.5780 & 31.1306 & 34.1731 & 38.6439 \\
\hline & $(r=3.0$ & $N=7$ & 15.9440 & 22.9208 & 26.5374 & 30.9030 & 34.0434 & 38.4578 \\
\hline & & $N=8$ & 15.9438 & 22.9202 & 26.5348 & 30.8917 & 33.9053 & 38.4258 \\
\hline & & $N=9$ & 15.9438 & 22.9202 & 26.5342 & 30.8896 & 33.8958 & 38.3850 \\
\hline & Reference [40] & - & 15.94 & 22.92 & 26.53 & 30.89 & 33.89 & 38.38 \\
\hline
\end{tabular}

and they all have a common number of components

$$
p=q=r=N N
$$

the matrix $[\mathbf{K}]$ has the structure of

$$
[\mathbf{K}]=\left[\begin{array}{ccc}
{\left[\mathbf{K}_{c c}\right]} & {\left[\mathbf{K}_{c d}\right]} & {\left[\mathbf{K}_{c e}\right]} \\
& {\left[\mathbf{K}_{d d}\right]} & {\left[\mathbf{K}_{d e}\right]} \\
\text { Symmetric } & & {\left[\mathbf{K}_{e e}\right]}
\end{array}\right]
$$


Table VII. Impact study of parameter $r$ on triangular plates with $\mathrm{S} * \mathrm{~S} * \mathrm{~S} *$ boundary conditions with apex angle $\beta=90^{\circ}, h / b=0.15$ and $N=10$ for Gauss' kernel and $N=9$ for Fejer's kernel.

\begin{tabular}{|c|c|c|c|c|c|c|c|}
\hline \multirow[b]{2}{*}{ Kernel } & \multirow{2}{*}{$\begin{array}{l}\text { Parameter of } \\
\text { grid points }\end{array}$} & \multicolumn{6}{|c|}{ Mode sequence number } \\
\hline & & 1 & 2 & 3 & 4 & 5 & 6 \\
\hline \multirow[t]{11}{*}{ Gauss } & $r=1.0$ & 11.2942 & 19.2944 & 23.2819 & 28.1813 & 31.2322 & 36.2219 \\
\hline & $r=1.2$ & 11.2787 & 19.2803 & 23.2763 & 28.1640 & 31.2255 & 36.2189 \\
\hline & $r=1.4$ & 11.2754 & 19.2750 & 23.2750 & 28.1561 & 31.2218 & 36.2174 \\
\hline & $r=1.6$ & 11.2748 & 19.2727 & 23.2745 & 28.1527 & 31.2197 & 36.2170 \\
\hline & $r=1.8$ & 11.2746 & 19.2715 & 23.2744 & 28.1511 & 31.2184 & 36.2176 \\
\hline & $r=2.0$ & 11.2744 & 19.2712 & 23.2743 & 28.1504 & 31.2187 & 36.2193 \\
\hline & $r=2.2$ & 11.2757 & 19.2710 & 23.2743 & 28.1496 & 31.2201 & 36.2193 \\
\hline & $r=2.4$ & 11.2753 & 19.2710 & 23.2690 & 28.1486 & 31.2194 & 36.2185 \\
\hline & $r=2.6$ & 11.2888 & 19.2701 & 23.2744 & 28.1537 & 31.2796 & 36.2318 \\
\hline & $r=2.8$ & - & - & - & - & - & - \\
\hline & $r=3.0$ & - & - & - & - & - & - \\
\hline Fejer & $r=1.0$ & - & - & - & - & - & - \\
\hline \multirow[t]{10}{*}{$(k=2)$} & $r=1.2$ & - & - & - & - & - & - \\
\hline & $r=1.4$ & - & - & - & - & - & - \\
\hline & $r=1.6$ & 12.0234 & 19.3896 & 23.4293 & 28.3548 & 31.2846 & 36.6767 \\
\hline & $r=1.8$ & 11.4122 & 19.2952 & 23.3038 & 28.1863 & 31.2368 & 36.3158 \\
\hline & $r=2.0$ & 11.2889 & 19.2777 & 23.2827 & 28.1621 & 31.2221 & 36.2448 \\
\hline & $r=2.2$ & 11.2763 & 19.2738 & 23.2775 & 28.1567 & 31.2210 & 36.2483 \\
\hline & $r=2.4$ & 11.2748 & 19.2725 & 23.2756 & 28.1535 & 31.2253 & 36.2591 \\
\hline & $r=2.6$ & 11.2746 & 19.2718 & 23.2749 & 28.1520 & 31.2316 & 36.2694 \\
\hline & $r=2.8$ & 11.2746 & 19.2715 & 23.2749 & 28.1520 & 31.2386 & 36.2781 \\
\hline & $r=3.0$ & 11.2757 & 19.2740 & 23.2797 & 28.1580 & 31.2511 & 36.2927 \\
\hline Reference [50] & - & 11.28 & 19.27 & 23.28 & 28.16 & 31.24 & 36.28 \\
\hline
\end{tabular}

Table VIII. Impact study of parameter $k$ on triangular plates with $\mathrm{S} * \mathrm{~S} * \mathrm{~S} *$ boundary conditions with $a / b=1.0, h / b=0.1$ and $N=9$.

\begin{tabular}{|c|c|c|c|c|c|c|c|}
\hline \multirow[b]{2}{*}{ Kernel } & \multirow{2}{*}{$\begin{array}{l}\text { Parameter of } \\
\text { grid points }\end{array}$} & \multicolumn{6}{|c|}{ Mode sequence number } \\
\hline & & 1 & 2 & 3 & 4 & 5 & 6 \\
\hline Fejer & $k=1$ & - & - & - & - & - & - \\
\hline \multirow[t]{9}{*}{$(r=3.0)$} & $k=2$ & 11.2757 & 19.2740 & 23.2797 & 28.1580 & 31.2511 & 36.2927 \\
\hline & $k=3$ & 11.2746 & 19.2716 & 23.2748 & 28.1522 & 31.2362 & 36.2750 \\
\hline & $k=4$ & 11.2746 & 19.2718 & 23.2749 & 28.1527 & 31.2338 & 36.2713 \\
\hline & $k=5$ & 11.2746 & 19.2719 & 23.2748 & 28.1523 & 31.2326 & 36.2698 \\
\hline & $k=6$ & 11.2746 & 19.2720 & 23.2748 & 28.1523 & 31.2319 & 36.2689 \\
\hline & $k=7$ & 11.2746 & 19.2720 & 23.2754 & 28.1524 & 31.2316 & 36.2683 \\
\hline & $k=8$ & 11.2746 & 19.2720 & 23.2748 & 28.1524 & 31.2314 & 36.2680 \\
\hline & $k=9$ & 11.2746 & 19.2720 & 23.2748 & 28.1524 & 31.2312 & 36.2677 \\
\hline & $k=10$ & 11.2746 & 19.2720 & 23.2748 & 28.1524 & 31.2314 & 36.2680 \\
\hline Reference [50] & - & 11.28 & 19.27 & 23.28 & 28.16 & 31.24 & 36.28 \\
\hline
\end{tabular}


Table IX. Comparison study for triangular plates with $\mathrm{S}^{*} \mathrm{CC}$ boundary conditions.

\begin{tabular}{|c|c|c|c|c|c|c|c|c|c|}
\hline \multirow[b]{2}{*}{$\beta^{\circ}$} & \multirow[b]{2}{*}{$h / b$} & \multirow[b]{2}{*}{ Kernel } & \multirow{2}{*}{$\begin{array}{c}\text { Parameter of } \\
\text { kernel }\end{array}$} & \multicolumn{6}{|c|}{ Mode sequence number } \\
\hline & & & & 1 & 2 & 3 & 4 & 5 & 6 \\
\hline \multirow[t]{9}{*}{$30^{\circ}$} & \multirow[t]{3}{*}{0.10} & Gauss & $r=1.8$ & 6.619 & 10.35 & 13.79 & 14.33 & 18.55 & 18.94 \\
\hline & & Fejer & $r=3.0$ & 6.617 & 10.35 & 13.78 & 14.33 & 18.64 & 18.94 \\
\hline & & Reference [40] & - & 6.617 & 10.35 & 13.78 & 14.33 & 18.62 & 18.94 \\
\hline & \multirow[t]{3}{*}{0.15} & Gauss & $r=1.8$ & 5.857 & 8.882 & 11.50 & 11.99 & 15.19 & 15.40 \\
\hline & & Fejer & $r=3.0$ & 5.857 & 8.883 & 11.50 & 11.99 & 15.23 & 15.41 \\
\hline & & Reference [40] & - & 5.857 & 8.883 & 11.49 & 11.99 & 15.22 & 15.41 \\
\hline & \multirow[t]{3}{*}{0.20} & Gauss & $r=1.8$ & 5.151 & 7.637 & 9.671 & 10.13 & 12.66 & 12.77 \\
\hline & & Fejer & $r=3.0$ & 5.150 & 7.636 & 9.673 & 10.13 & 12.68 & 12.77 \\
\hline & & Reference [40] & - & 5.151 & 7.638 & 9.671 & 10.13 & 12.68 & 12.77 \\
\hline \multirow[t]{9}{*}{$60^{\circ}$} & \multirow[t]{3}{*}{0.10} & Gauss & $r=1.6$ & 10.62 & 19.20 & 19.44 & 28.24 & 29.78 & 29.81 \\
\hline & & Fejer & $r=3.0$ & 10.62 & 19.20 & 19.44 & 28.24 & 29.77 & 29.81 \\
\hline & & Reference [40] & - & 10.63 & 19.20 & 19.44 & 28.24 & 29.78 & 29.81 \\
\hline & \multirow[t]{3}{*}{0.15} & Gauss & $r=1.6$ & 9.044 & 15.48 & 15.80 & 22.11 & 23.21 & 23.26 \\
\hline & & Fejer & $r=3.0$ & 9.045 & 15.48 & 15.80 & 22.12 & 23.22 & 23.26 \\
\hline & & Reference [40] & - & 9.045 & 15.48 & 15.80 & 22.12 & 23.22 & 23.26 \\
\hline & \multirow[t]{3}{*}{0.20} & Gauss & $r=1.6$ & 7.722 & 12.75 & 13.09 & 17.93 & 18.78 & 18.83 \\
\hline & & Fejer & $r=3.0$ & 7.722 & 12.75 & 13.09 & 17.93 & 18.78 & 18.83 \\
\hline & & Reference [40] & - & 7.724 & 12.75 & 13.10 & 17.93 & 18.78 & 18.83 \\
\hline \multirow[t]{9}{*}{$90^{\circ}$} & \multirow[t]{3}{*}{0.10} & Gauss & $r=1.2$ & 17.28 & 27.26 & 33.08 & 38.99 & 43.56 & 50.91 \\
\hline & & Fejer & $r=3.0$ & 17.28 & 27.26 & 33.08 & 38.98 & 43.57 & 50.93 \\
\hline & & Reference [40] & - & 17.28 & 27.26 & 33.08 & 38.98 & 43.57 & 50.93 \\
\hline & \multirow[t]{3}{*}{0.15} & Gauss & $r=1.2$ & 14.00 & 21.13 & 25.46 & 29.40 & 32.72 & 37.86 \\
\hline & & Fejer & $r=3.0$ & 14.00 & 21.13 & 25.47 & 29.40 & 32.72 & 37.88 \\
\hline & & Reference [40] & - & 14.00 & 21.23 & 25.47 & 29.40 & 32.72 & 37.88 \\
\hline & \multirow[t]{3}{*}{0.20} & Gauss & $r=1.2$ & 11.55 & 17.01 & 20.45 & 23.32 & 25.89 & 28.73 \\
\hline & & Fejer & $r=3.0$ & 11.55 & 17.00 & 20.45 & 23.32 & 25.89 & 28.73 \\
\hline & & Reference [40] & - & 11.55 & 17.01 & 20.45 & 23.32 & 25.89 & 28.74 \\
\hline
\end{tabular}

and similarly the matrix $[\mathbf{M}]$ is given by

$$
[\mathbf{M}]=\left[\begin{array}{ccc}
{\left[\mathbf{M}_{c c}\right]} & {\left[\mathbf{M}_{c d}\right]} & {\left[\mathbf{M}_{c e}\right]} \\
& {\left[\mathbf{M}_{d d}\right]} & {\left[\mathbf{M}_{d e}\right]} \\
\text { Symmetric } & & {\left[\mathbf{M}_{e e}\right]}
\end{array}\right]
$$

where the elements of $[\mathbf{K}]$ are given by

$$
K_{c c i j}=\frac{b}{a} \kappa G h \int_{\bar{A}} \frac{\partial \phi_{i}}{\partial \xi} \frac{\partial \phi_{j}}{\partial \xi} \mathrm{d} \bar{A}+\frac{a}{b} \kappa G h \int_{\bar{A}} \frac{\partial \phi_{i}}{\partial \eta} \frac{\partial \phi_{j}}{\partial \eta} \mathrm{d} \bar{A}
$$


Table X. Comparison study for triangular plates with CFF boundary conditions.

\begin{tabular}{|c|c|c|c|c|c|c|c|c|c|}
\hline \multirow[b]{2}{*}{$\beta^{\circ}$} & \multirow[b]{2}{*}{$h / b$} & \multirow[b]{2}{*}{ Kernel } & \multirow{2}{*}{$\begin{array}{l}\text { Parameter of } \\
\text { kernel }\end{array}$} & \multicolumn{6}{|c|}{ Mode sequence number } \\
\hline & & & & 1 & 2 & 3 & 4 & 5 & 6 \\
\hline \multirow[t]{9}{*}{$30^{\circ}$} & \multirow[t]{3}{*}{0.10} & Gauss & $r=1.8$ & 0.3117 & 1.326 & 1.980 & 3.148 & 4.726 & 5.648 \\
\hline & & Fejer & $r=3.0$ & 0.3115 & 1.326 & 1.981 & 3.148 & 4.727 & 5.647 \\
\hline & & Reference [40] & - & 0.3115 & 1.326 & 1.980 & 3.148 & 4.725 & 5.647 \\
\hline & \multirow[t]{3}{*}{0.15} & Gauss & $r=1.8$ & 0.3092 & 1.292 & 1.852 & 2.995 & 4.272 & 5.223 \\
\hline & & Fejer & $r=3.0$ & 91 & 1.292 & 1.852 & 2.995 & 272 & 5.223 \\
\hline & & Reference [40] & - & 0.3091 & 1.292 & 1.851 & 2.995 & 4.272 & 5.223 \\
\hline & \multirow{3}{*}{0.20} & Gauss & $r=1.8$ & 0.3063 & 1.251 & 1.709 & 2.820 & 3.800 & 4.780 \\
\hline & & Fejer & $=3.0$ & 0.3063 & 1.251 & 1.709 & 2.820 & .801 & 4.780 \\
\hline & & Reference [40] & - & 0.3062 & 1.251 & 1.709 & 2.820 & 3.801 & 4.780 \\
\hline \multirow[t]{9}{*}{$60^{\circ}$} & \multirow[t]{3}{*}{0.10} & Gauss & $r=1.8$ & 1.376 & 5.000 & 5.540 & 12.01 & 12.12 & 13.85 \\
\hline & & Fejer & $r=3.0$ & 376 & 5.000 & 5.540 & 12.01 & 12.12 & 13.85 \\
\hline & & Reference [40] & - & 1.376 & 4.999 & 5.540 & 12.00 & 12.12 & 13.85 \\
\hline & \multirow[t]{3}{*}{0.15} & Gauss & $r=1.8$ & 1.339 & 4.529 & 5.066 & 10.34 & 10.43 & 11.77 \\
\hline & & Fejer & $r=3.0$ & 9 & 4.530 & 5.066 & 10.34 & 10.43 & 11.77 \\
\hline & & Reference [40] & - & 1.339 & 4.529 & 5.066 & 10.34 & 10.43 & 11.77 \\
\hline & \multirow[t]{3}{*}{0.20} & Gauss & $r=1.8$ & 1.295 & 4.051 & 4.586 & 8.801 & 9.059 & 10.05 \\
\hline & & Feje & $=3.0$ & 1 & 4.051 & 4.586 & 8.802 & 9.0 & \\
\hline & & Reference [40] & - & 1.295 & 4.051 & 4.586 & 8.802 & 9.060 & 10.06 \\
\hline \multirow[t]{9}{*}{$90^{\circ}$} & \multirow[t]{3}{*}{0.10} & Gauss & $r=1.8$ & 3.74 & 9.661 & 13.41 & 20.10 & 22.19 & 27.95 \\
\hline & & Fejer & $r=3.0$ & 3.7410 & 9.661 & 13.41 & 20.11 & 22.19 & 27.95 \\
\hline & & Reference [40] & - & 3.741 & 9.661 & 13.41 & 20.11 & 22.19 & 27.96 \\
\hline & \multirow[t]{3}{*}{0.15} & Gauss & $r=1.8$ & 3.511 & 8.391 & 11.31 & 16.43 & 17.96 & 21.96 \\
\hline & & Fejer & $r=3.0$ & 3.511 & 8.392 & 11.31 & 16.43 & 17.96 & 21.96 \\
\hline & & Reference [40] & - & 3.511 & 8.392 & 11.31 & 16.43 & 17.96 & 21.96 \\
\hline & \multirow[t]{3}{*}{0.20} & Gauss & $r=1.8$ & 3.266 & 7.249 & 9.582 & 13.65 & 14.81 & 17.84 \\
\hline & & Fejer & $r=3$. & & & 9.582 & 13.65 & 14.81 & 17.84 \\
\hline & & Reference [40] & - & 3.267 & 7.250 & 9.583 & 13.65 & 14.81 & 17.85 \\
\hline
\end{tabular}

$$
\begin{aligned}
K_{c d i j} & =b \kappa G h \int_{\bar{A}} \frac{\partial \phi_{i}}{\partial \xi} \psi_{x j} \mathrm{~d} \bar{A} \\
K_{c e i j} & =a \kappa G h \int_{\bar{A}} \frac{\partial \phi_{i}}{\partial \eta} \psi_{y j} \mathrm{~d} \bar{A} \\
K_{d d i j} & =\frac{b}{a} D \int_{\bar{A}} \frac{\partial \psi_{x i}}{\partial \xi} \frac{\partial \psi_{x j}}{\partial \xi} \mathrm{d} \bar{A}+\frac{a}{b} \frac{D(1-v)}{2} \int_{\bar{A}} \frac{\partial \psi_{x i}}{\partial \eta} \frac{\partial \psi_{x j}}{\partial \eta} \mathrm{d} \bar{A}+a b \kappa G h \int_{\bar{A}} \psi_{x i} \psi_{x j} \mathrm{~d} \bar{A} \\
K_{d e i j} & =v D \int_{\bar{A}} \frac{\partial \psi_{x i}}{\partial \xi} \frac{\partial \psi_{y j}}{\partial \eta} \mathrm{d} \bar{A}+\frac{D(1-v)}{2} \int_{\bar{A}} \frac{\partial \psi_{x i}}{\partial \eta} \frac{\partial \psi_{x j}}{\partial \xi} \mathrm{d} \bar{A}
\end{aligned}
$$




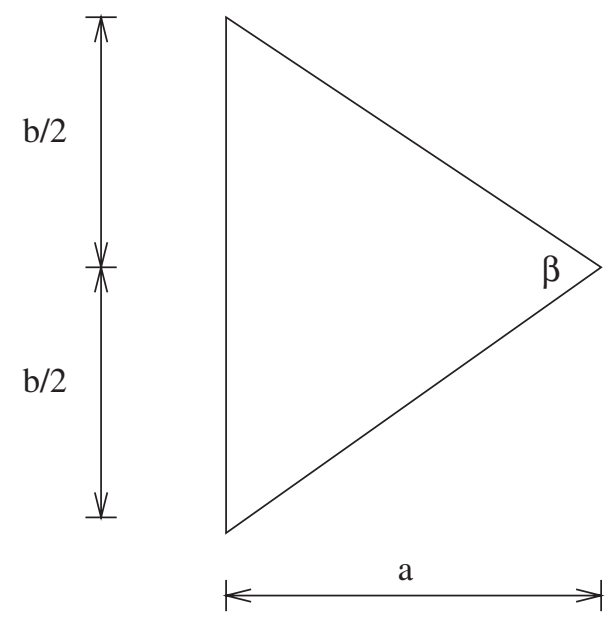

Figure 6. Geometry of a triangular Mindlin plate.
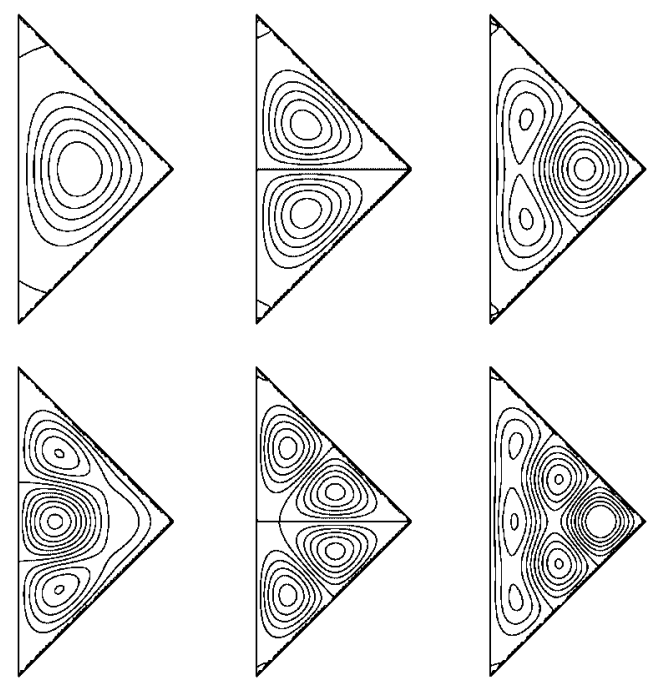

Figure 7. First six mode shapes of a CCC right angled isosceles triangular plate with base length $b(h / b=0.15)$.

$$
K_{e e i j}=\frac{a}{b} D \int_{\bar{A}} \frac{\partial \psi_{y i}}{\partial \eta} \frac{\partial \psi_{y j}}{\partial \eta} \mathrm{d} \bar{A}+\frac{b}{a} \frac{D(1-v)}{2} \int_{\bar{A}} \frac{\partial \psi_{y i}}{\partial \xi} \frac{\partial \psi_{y j}}{\partial \xi} \mathrm{d} \bar{A}+a b \kappa G h \int_{\bar{A}} \psi_{y i} \psi_{y j} \mathrm{~d} \bar{A}
$$

where

$$
i=1,2, \ldots, p ; \quad j=1,2, \ldots, p
$$



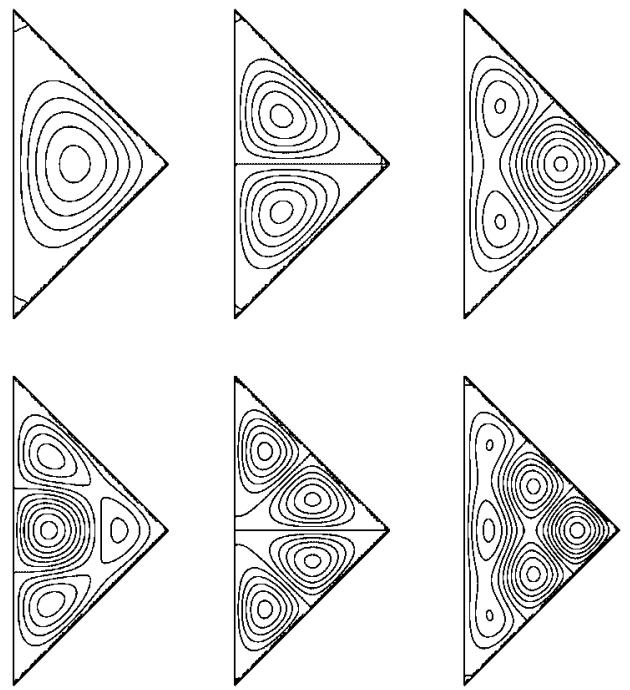

Figure 8. First six mode shapes of an $S * S * S *$ right angled isosceles triangular plate with base length $b(h / b=0.15)$.
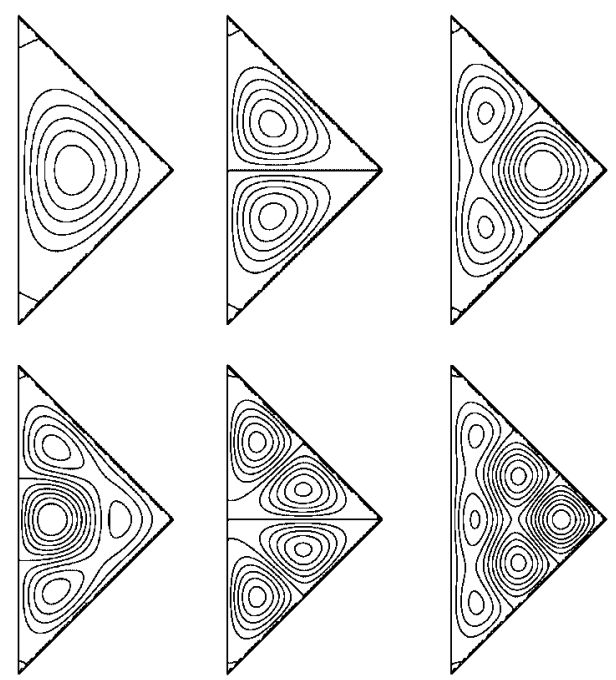

Figure 9. First six mode shapes of an $\mathrm{S} * \mathrm{CC}$ right angled isosceles triangular plate with base length $b(h / b=0.15)$.

for all of the above-mentioned elements. Finally, the entries of $[\mathbf{M}]$ are given by

$$
M_{c c i j}=a b \rho h \int_{\bar{A}} \phi_{i} \phi_{j} \mathrm{~d} \bar{A}
$$



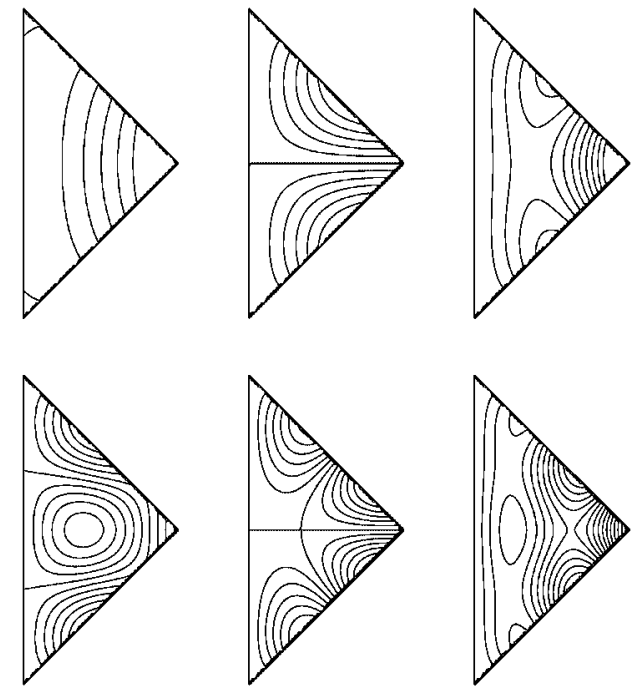

Figure 10. First six mode shapes of a CFF right angled isosceles triangular plate with base length $b(h / b=0.15)$.

$$
\begin{aligned}
M_{c d i j} & =0 \\
M_{c e i j} & =0 \\
M_{d d i j} & =\frac{1}{12} a b \rho h^{3} \int_{\bar{A}} \psi_{x i} \psi_{x j} \mathrm{~d} \bar{A} \\
M_{d e i j} & =0 \\
M_{e e i j} & =\frac{1}{12} a b \rho h^{3} \int_{\bar{A}} \psi_{y i} \psi_{y j} \mathrm{~d} \bar{A}
\end{aligned}
$$

where

$$
i=1,2, \ldots, p ; \quad j=1,2, \ldots, p
$$

and for simplicity, we choose $p=N \times N$ in this work.

For vibration analysis, our objective is to obtain the frequency parameter, $\omega$, which can be accomplished by solving the generalized eigenvalue problem defined by Equation (33). We resort to a standard eigenvalue solver for the solution of Equation (33).

\section{NUMERICAL RESULTS AND DISCUSSION}

In this section, we explore the usefulness and test the accuracy of the DSC-Ritz method. Consideration is given to the first six frequency parameters for thick rectangular and isosceles 
triangular plates with different combinations of simply supported, clamped and free edges. Note that a simply supported edge for the rectangular plate is considered to be the first type (S) and for the triangular plates is treated as the second type $\left(\mathrm{S}^{*}\right)$ in the paper. Numerical integrations are carried out by using Gaussian quadratures with appropriate number of polynomials (about 50).

\subsection{Rectangular plates}

For brevity and convenience, a four-letter symbol is used to denote the support conditions of a rectangular Mindlin plate. For example, an SCFS plate has a simply supported left edge, a clamped bottom edge, a free right edge and a simply supported top edge, respectively. For the purpose of comparison study, numerical calculations have been performed for rectangular Mindlin plates of four different combinations of edge support conditions, namely CCCC, SSSS, CSSF, and CFSF plates. The vibration frequencies of a rectangular Mindlin plate are expressed in terms of a non-dimensional frequency parameter $\lambda=\left(\omega b^{2} / \pi^{2}\right) \sqrt{\rho h / D}$, where $b$ is the width of the plate.

The Poisson's ratio $v=0.3$ and the shear correction factor $\kappa=\frac{5}{6}$ have been used in the calculation.

Convergence studies have been carried out for the CCCC plate. By varying the number of uniform DSC grid points $N$ both in $x$ and $y$ directions, the convergence patterns of the first six frequency parameters have been investigated. The results are summarized in Table I for the cases of thick rectangular plates with plate thickness ratios $h / b=0.1$ and 0.2 , plate aspect ratios $a / b=1.0$ and 2.0, and $r=1.8$ for the Gauss kernel and $r=3.0$ and $k=2$ for the Fejer kernel, respectively. The $p b-2$ Ritz results [50] have also been given in Table I for a comparison.

Table I shows that the frequency parameters decrease as the number of DSC grid points varies from 2 to 7 . For most cases, the frequency parameters for the CCCC plate have achieved good convergence even with the number of DSC grid points $N=6$. When the number of grid points $N$ increase from 6 to 9 , the frequency parameters oscillate slightly around those of the reference $p b-2$ Ritz. It is evident from the convergence studies that, in general, when the number of DSC grid points is set to be $N=9$ for both Gauss' kernel and Fejer's kernel, the DSC-Ritz method will produce accurate and reliable frequency parameters for rectangular Mindlin plates. All subsequent calculations for rectangular Mindlin plates are based on $N=9$.

Tables II and III show the impact of kernel parameters $r$ and $k$ on the frequency parameters of SSSS rectangular plates. It is evident that the DSC-Ritz method is robust against parameter variation. The selection of $r=1.8$ for the Gauss kernel and $r=3.0$ and $k=2$ for the Fejer kernel is appropriate for the present calculation.

These convergence and impact studies have established our confidence on the robustness and reliability of the DSC-Ritz method. To further explore the application of the method to rectangular plates with different combinations of edge support conditions, the first six frequency parameters for CSSF and CFSF rectangular Mindlin plates are computed with the Gauss kernel and the Fejer kernel and presented in Tables IV and V. The plate thickness is set to be $h / b=0.1$ and 0.2 and the plate aspect ratio is taken as $a / b=0.6,1.0,1.5,2.0$ and 2.5, respectively. The $p b-2$ Ritz results from Reference [50] are also presented in Tables IV and V. Excellent agreement is observed between the DSC-Ritz and $p b-2$ Ritz frequency parameters for all considered cases. Such agreement confirms the validity of the proposed DSC-Ritz method for vibration analysis of rectangular Mindlin plates. 
Figures 2-5 present the mode contour shapes for the first six modes of the CCCC, SSSS, CSSF, and CFSF square plates with plate thickness $h / b=0.10$. We observe that the mode shapes for square Mindlin plates of various edge support conditions are correctly predicted by the DSC-Ritz method.

\subsection{Isosceles triangular plates}

To verify the validity and versatility of the DSC-Ritz method for the analysis of plates of other geometries, we apply it to the determination of the first six vibration frequency parameters

$\lambda=\left(\omega b^{2} / 2 \pi\right) \sqrt{\rho h / D}$ for thick isosceles triangular plates as shown in Figure 6. Coordinate transformation is similar to that in Reference [50]. Uniform reference meshes are used in the computational domain. For the purpose of comparison study, numerical calculations have been performed for isosceles triangular plates with different apex angles $(\beta=30,60$ and $\left.90^{\circ}\right)$, thickness ratios $(h / b=0.10,0.15$ and 0.20$)$ and four different combinations of boundary conditions $(S * S * S *, C C C, S * C C, C F F)$. Note that the three-letter symbol used to describe the plate edge support conditions refers to the left edge, the lower inclined edge and the upper inclined edge, respectively. The Poisson ratio $v=0.3$ and the shear correction factor $\kappa=\pi^{2} / 12$ are used in the calculation.

Convergence studies are carried out for the $\mathrm{CCC}$ isosceles triangular plates with the apex angle $\beta=30,60$ and $90^{\circ}$, and the thickness ratio $h / b=0.15$. The results obtained by the DSC-Ritz method are presented in Table VI with different numbers of DSC grid points $N$. The $p b$-2 Ritz solutions [40] are also presented in Table VI. Unlike the convergence cases for rectangular Mindlin plates where the frequency parameters oscillates slightly as the number of DSC grid points $N$ increases from 6 to 9, the frequency parameters for the triangular Mindlin plates decrease monotonically as $N$ increases. It is evident from the convergence studies that in general $N=10$ is required for Gauss kernel and $N=9$ is required for Fejer kernel to get accurate solutions. Thus, all vibration results determined herein are based on $N=10$ for the Gauss kernel and $N=9$ for the Fejer kernel, respectively.

Tables VII and VIII show the impact of kernel parameters $r$ and $k$ on the frequency parameters of $S * S^{*} S *$ triangular plates. It is seen that results are more sensitive to the local influence domain parameter $r$ than to the parameter $k$. However, for both Gauss and Fejer kernels, there are a wide range of $r$ values that give correct results judged by the literature [40]. We choose $k=2$ in the rest of the work. The value of $r$ is specified in all computations. Certainly, a detailed study of the impact of the parameter space as that given by Driscoll and Fornberg for their radial basis functions [51] would aid our understanding. This issue will be addressed elsewhere.

Tables IX and $\mathrm{X}$ present the comparison studies of the first six frequency parameters for $\mathrm{S} * \mathrm{CC}$ and $\mathrm{CFF}$ isosceles triangular plates. It can be seen that the results are all in good agreement with those in Reference [40].

Figures 7-10 show the mode contour shapes for four right angled isosceles triangular Mindlin plates with thickness ratio $h / b=0.15$. Again, the DSC-Ritz method is able to predict the correct vibration mode shapes for triangular Mindlin plates.

\section{CONCLUSION}

This paper introduces a novel numerical method, the DSC-Ritz method, for the vibration analysis of thick plates based on the Mindlin first-order shear deformable plate theory. The key 
idea is to take the advantage of DSC local delta sequence kernels and the $p b$-2 Ritz boundary functions. Two DSC delta sequence kernels of positive type are employed to construct new basis functions. The Ritz variational principle is utilized to determine unknown expansion coefficients and to arrive at a set of generalized eigenvalue equations. The solution of the generalized eigenvalue equations results in the desirable frequency parameters and modal shapes. Numerical experiments are conducted for rectangular plates and triangular plates with various combinations of simply supported, clamped and free edge conditions. The reliability and robustness of the proposed method are carefully validated by extensive convergence tests and by a comparison with those in the literature. Numerical results indicate that the proposed DSC-Ritz method is a simple approach for the vibration analysis of Mindlin plates.

Comparing to our previous DSC algorithm, which makes use of delta sequence kernels of Dirichlet type and the collocation formulation for differential equations, the present DSCRitz method employs delta sequence kernels of positive type and is relied on Ritz energy minimization principle (essentially the Galerkin formulation). Obviously, the philosophy of discrete approximations to the singular delta distribution, i.e. the universal reproducing kernel, underpins both DSC methods. We believe that these DSC methods are promising new approaches for structural analysis in general.

\section{REFERENCES}

1. Chladni EFF. Entdeckungen uber die Theorie des Klanges. Weidmanns Erben \& Reich: Leipzig, 1787.

2. Kirchhoff G. Uber das gleichgwich und die bewegung einer elastischen scheibe. Journal of Angew Mathematics $1850 ; 40: 51-88$.

3. Reissner E. The effect of transverse shears deformation on the bending of elastic plate. Journal of Applied Mechanics (ASME) 1945; 12:69-76.

4. Mindlin RD. Influence of rotary inertia and shear in flexural motion of isotropic, elastic plates. Journal of Applied Mechanics (ASME) 1951; 18:1031-1036.

5. Leissa AW. Vibration of Plates (NASA SP 160). U.S. Government Printing Office: Washington DC, 1969.

6. Gorman DJ. Highly accurate analytical solution for free vibration analysis of simply supported right triangular plates. Journal of Sound and Vibration 1983; 89:107-118.

7. Warburton GB. The vibration of rectangular plates. Proceedings of the Institute of Mechanical Engineering 1954; 168:371-384.

8. Young D. Vibration of rectangular plates by the Ritz method. Journal of Applied Mechanics (ASME) 1950; 17:448-453.

9. Durvasula S. Nature frequencies and modes of skew membranes. Journal of Acoustical Society of America 1968; 44:1636-1646.

10. Leissa AW. The free vibration of rectangular plates. Journal of Sound Vibration 1973; 31:257-293.

11. Mizusawa T. Natural frequencies of rectangular plates with free edges. Journal of Sound and Vibration 1986; 105:451-459.

12. Martin AF, Leissa AW. Application of the Ritz method to plane elasticity problems for composite sheets with variable fiber spacing. International Journal for Numerical Methods in Engineering 1989; 28:1813-1825.

13. Wang S. A unified Timoshenko beam B-spline Rayleigh-Ritz method for vibration and buckling analysis of thick and thin beams and plates. International Journal for Numerical Methods in Engineering 1997; 40:473-491.

14. Smith ST, Bradford MA, Oehlers D. Numerical convergence of simple and orthogonal polynomials for the unilateral plate buckling problem using the Rayleigh-Ritz method. International Journal for Numerical Methods in Engineering 1999; 44:1685-1707.

15. Narita Y. Application of a series-type method to vibration of orthotropic rectangular plates with mixed boundary conditions. Journal of Sound and Vibration 1981; 77:345-355.

16. Laura PAA, Gutierrez RH. Analysis of vibrating rectangular plates with nonuniform boundary conditions by using the differential quadrature method. Journal of Sound and Vibration 1994; 173:702-706. 
17. Shu C, Wang CM. Implementation of clamped and simply supported boundary conditions in the GDQ free vibration analysis of beams and plates. International Journal of Solids and Structures 1997; 34:819-835.

18. Shu C, Wang CM. Treatment of mixed and nonuniform boundary conditions in GDQ vibration analysis of rectangular plates. Engineering Structures 1999; 21:125-134.

19. Cheung YK, Cheung MS. Flexural vibration of rectangular and other polygonal plates. Proceedings of ASCE Journal of the Engineering Mechanics Division 1971; 97:391-411.

20. Fan SC, Cheung YK. Flexural free vibrations of rectangular plates with complex support conditions. Journal of Sound and Vibration 1984; 93:81-94.

21. Zienkiewicz OC. Achievements and some unsolved problems of the finite element method. International Journal for Numerical Methods in Engineering 2000; 47:9-28.

22. Wei GW. Discrete singular convolution for the solution of the Fokker-Planck equations. Journal of Chemical Physics 1999; 110:8930-8942.

23. Wei GW. A unified approach for the solution of the Fokker-Planck equations. Journal of Physics A: Mathematics and General 2000; 33:4935-4953.

24. Wei GW, Zhao YB, Xiang Y. Discrete singular convolution and its application to the analysis of plates with internal supports. I. Theory and algorithm. International Journal for Numerical Methods in Engineering 2002; 55:913-946.

25. Zhou YC, Patnaik BSV, Wan DC, Wei GW. DSC solution for flows in a staggered double lid driven cavity. International Journal for Numerical Methods in Engineering 2003; 57:211-234.

26. Bao G, Wei GW, Zhao S. Numerical solution of the Helmholtz equation with high wave numbers. International Journal for Numerical Methods in Engineering 2004; 59:389-408.

27. Wei GW. Vibration analysis by discrete singular convolution. Journal of Sound and Vibration 2001; 244: 535-553.

28. Wei GW. Discrete singular convolution for beam analysis. Engineering Structure 2001; 23:1045-1053.

29. Wei GW. A new algorithm for solving some mechanical problems. Computer Methods for Applied Mechanics and Engineering 2001; 190:2017-2030.

30. Wei GW, Zhao YB, Xiang Y. The determination of natural frequencies of rectangular plates with mixed boundary conditions by discrete singular convolution. International Journal of Mechanical Sciences 2001; 43:1731-1746.

31. Wei GW, Zhao YB, Xiang Y. A novel approach for the prediction and control of high frequency vibration. Journal of Sound and Vibration 2002; 257:207-246.

32. Xiang Y, Zhao YB, Wei GW. Discrete singular convolution and its application to the analysis of plates with internal supports. II. Complex supports. International Journal for Numerical Methods in Engineering 2002; 55:947-971.

33. Zhao YB, Wei GW. DSC analysis rectangular plates with nonuniform boundary conditions. Journal of Sound and Vibration 2002; 255:203-225.

34. Zhao YB, Wei GW, Xiang Y. Discrete singular convolution for the prediction of high frequency vibration of plates. International Journal of Solids and Structures 2002; 39:65-68.

35. Zhao YB, Wei GW, Xiang Y. Plate vibration under irregular internal supports. International Journal of Solids and Structures 2002; 39:1361-1383.

36. Liew KM, Wang CM, Xiang Y, Kitipornchai S. Vibration of Mindlin Plate, Programming the p-version Method. Elsevier: Amsterdam, 1998.

37. Kitipornchai S, Xiang Y, Wang CM, Liew KM. Buckling of thick skew plates. International Journal for Numerical Methods in Engineering 1993; 36:1299-1310.

38. Dickinson SM, Li EKH. On the use of simply supported plate functions in the Rayleigh-Ritz method applied to the flexural vibration of triangular plates. Journal of Sound and Vibration 1982; 80:292-297.

39. Nanni J. Das Eulersche Knickproblem unter beruksichtigung der querkrafte. Zeitschrift fur Angewandte Mathematik and Physik 1971; 22:156-185.

40. Kitipornchai S, Xiang Y, Wang CM, Liew KM. Free vibration of isosceles triangular Mindlin plates. International Journal of Mechanical Sciences 1993; 35:89-102.

41. Liew KM, Xiang Y, Wang CM, Kitipornchai S. Flexural vibration of shear deformable circular and annular plates on ring supports. Computer Methods in Applied Mechanics and Engineering 1993; 110:301-315.

42. Xiang Y, Wang CM, Kitipornchai S. Buckling of skew Mindlin plates subjected to in-plane shear loadings. International Journal of Mechanical Sciences 1995; 37:1089-1101.

43. Xiang Y, Wang CM, Kitipornchai S. FORTRAN subroutines for mathematical operations on polynomial functions. Computers and Structures 1995; 56:541-551. 
44. Wei GW, Xiang Y. DSC-Ritz method for the analysis of beams and plates, 2003, to be published.

45. Lucy LB. A numerical approach to the testing of the fission hypothesis. The Astrophysical Journal 1977; 82:1013-1024.

46. Monaghan JJ. An introduction to SPH. Computer Physics Communication 1988; 48:89-96.

47. Liu WK, Jun S, Li S, Adee J, Belytschko T. Reproducing kernel particle method for structural dynamics. International Journal for Numerical Methods in Engineering 1995; 38:1655-1679.

48. Liew KW, Zhao X, Ng TN. The element-free $k p$-Ritz method for vibration of laminated rotating cylindrical panels. International Journal of Structural Stability and Dynamics 2002; 2:523-558.

49. Zhao X, Liew $\mathrm{KW}, \mathrm{Ng} \mathrm{TN}$. Vibration analysis of laminated composite cylindrical panels via a meshfree approach. International Journal of Solids and Structures 2003; 40:161-180.

50. Liew KM, Xiang Y, Kitipornchai S. Transverse vibration of thick rectangular plates-I: comprehensive sets of boundary conditions. Computers and Structures 1993; 49:1-29.

51. Driscoll TA, Fornberg B. Interpolation in the limit of increasingly flat radial basis functions. Computers and Mathematics with Applications 2002; 43:413-422. 